PALEO

Revue d'archéologie préhistorique

$23 \mid 2012$

Varia

\title{
Des apprentis gravettiens ont-ils confectionné des armatures lithiques à Tercis (Landes, France)?
}

Aurélien Simonet

(2) OpenEdition

Journals

Édition électronique

URL : http://journals.openedition.org/paleo/2456

DOI : $10.4000 /$ paleo.2456

ISSN : 2101-0420

Éditeur

SAMRA

Édition imprimée

Date de publication : 15 décembre 2012

Pagination : 249-276

ISSN : $1145-3370$

\section{Référence électronique}

Aurélien Simonet, « Des apprentis gravettiens ont-ils confectionné des armatures lithiques à Tercis (Landes, France) ? », PALEO [En ligne], 23 | 2012, mis en ligne le 19 avril 2013, consulté le 25 juillet 2020. URL : http://journals.openedition.org/paleo/2456 ; DOI : https://doi.org/10.4000/paleo.2456

Ce document a été généré automatiquement le 25 juillet 2020

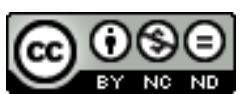

PALEO est mis à disposition selon les termes de la licence Creative Commons Attribution - Pas d'Utilisation Commerciale - Pas de Modification 4.0 International. 


\title{
Des apprentis gravettiens ont-ils confectionné des armatures lithiques à Tercis (Landes, France)?
}

\author{
Aurélien Simonet
}

\section{1 - Présentation du site}

\section{1 - Présentation géographique}

1 Le site archéologique de Tercis (Landes, France) est localisé au sud-ouest de Dax, sur le flanc sud d'un anticlinal, sur une colline qui domine l'Adour de 60 mètres environ (fig. 1). Il est constitué d'un ensemble de petites concentrations de silex récoltées en surface depuis le XIXè siècle. L'ensemble de ces concentrations se répartit d'ouest en est au sommet d'une crête qui se prolonge sur plus d'un kilomètre. Il paraît très probable que d'autres occupations restent à découvrir dans les secteurs non explorés. Le contexte géologique présente de nombreux affleurements de silex. Le site est, de fait, un gigantesque atelier de taille qui a servi de réserve de silex à de nombreux groupes humains du Paléolithique moyen et supérieur avant de laisser la place à une exploitation contemporaine du calcaire pour fabriquer de la chaux (Normand 1987, 1993 ; Kozlowski et Lenoir 1988 ; Kawalek 2008 ; Simonet 2004, 2009). Parmi ceux-ci, le Gravettien semble particulièrement bien représenté. D'ailleurs, les déterminations pétroarchéologiques au sein du Gravettien des grottes d'Isturitz et de Brassempouy, situées toutes les deux à une égale distance de Tercis (une trentaine de kilomètres), confirment l'utilisation importante du silex de Tercis notamment pour la confection des pointes à dos (Simonet 2009, 2010, 2012). À l'heure actuelle, Tercis représente l'atelier de taille connu le plus important, en relation avec les grands gisements d'Isturitz et de Brassempouy. Plus au nord, Montaut pourrait représenter un autre exemple de site satellite dédié à des activités de taille du silex, provenant cette fois de 
l'anticlinal d'Audignon, où des vestiges gravettiens ont notamment été identifiés (Merlet 1996). Plus au sud, la découverte récente du site de l'Avenue du Prissé à Bayonne représente un nouveau jalon gravettien où les activités de taille du silex jouaient un rôle prépondérant (Redondo 2011).

\section{2 - Présentation historiographique}

2 L'importance du site de Tercis, et de l'ensemble de la Chalosse en général, a été saisie très tôt, dès la fin du XIXè siècle par des érudits locaux (Daguin 1948 ; Du Boucher 1877, 1878, 1879 ; Pottier 1872 ). Il peut sembler d'autant plus paradoxal, de fait, que le site soit négligé par la suite, durant la plus grande partie de la seconde moitié du XXè siècle. Ainsi, les travaux de R. Arambourou (1963) et surtout la thèse de C. Thibault sur les terrains quaternaires du bassin de l'Adour (Thibault 1970) sont les seules études universitaires mentionnant le site de Tercis. C. Thibault donne des informations précises d'ordre stratigraphique et sédimentologique avec notamment la coupe du talus de Vignès. Il mentionne la richesse du site en nucléus à lames, en burins et en lames brutes et présente certaines pointes à dos de la collection Emile Daguin, réunies entre 1911 et 1920, qu'il attribue au Périgordien évolué. Plus récemment, ces mêmes pointes à dos de la collection Emile Daguin ont été étudiées selon des caractères morphologiques et morphométriques (Kozlowski et Lenoir 1988). Les données récentes sont entièrement l'œuvre du travail de $\mathrm{C}$. Normand qui collecta plusieurs séries rapportées à l'Aurignacien et au Gravettien et publia la série à pièces à dos (Normand 1987, 1993 ; Kawalek 2008 ; Simonet 2009).

Figure 1 - Localisation du site de Tercis par rapport aux grottes de Brassempouy, d'Isturitz et de Gargas, les sites gravettiens majeurs des Pyrénées.

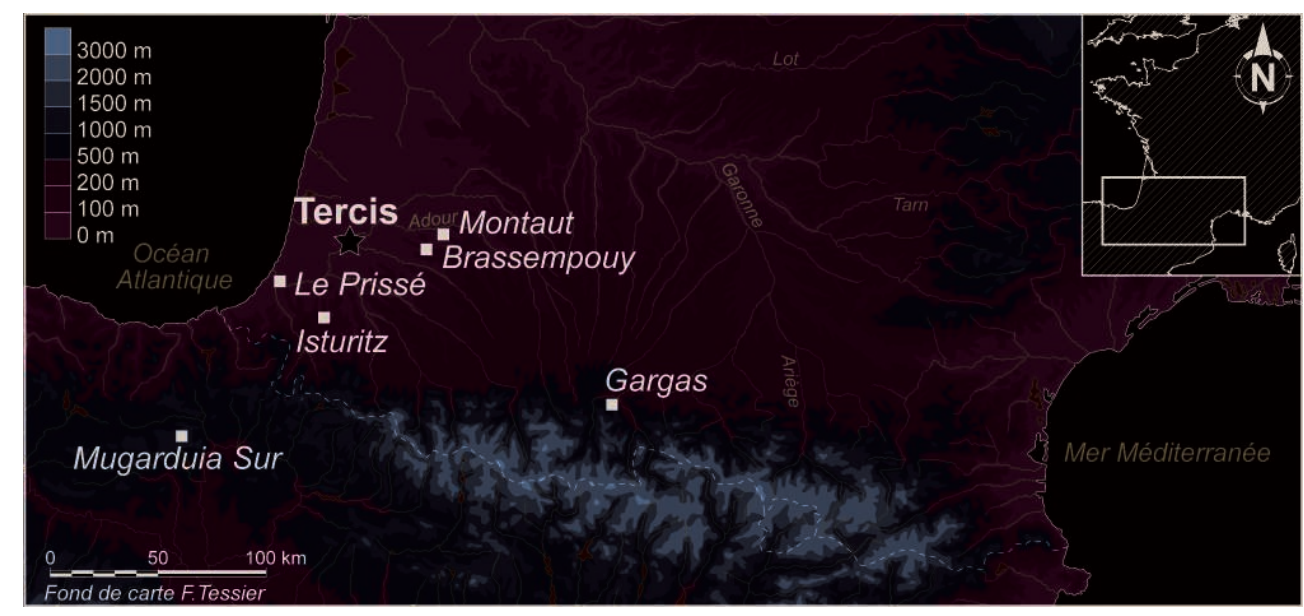

\section{3 - Présentation des trois principales séries gravettiennes}

3 La plupart des pièces archéologiques correspondent à des collectes de surface séparées de tout contexte stratigraphique et/ou archéologique et sont, par conséquent, difficilement exploitables. Concernant le Gravettien, trois séries, correspondant chacune en la collecte non exhaustive d'une concentration, sont particulièrement intéressantes malgré la perte d'une partie des données. Il s'agit, d'une part, de la série découverte par Emile Daguin près de la ferme des Vignaux, caractérisée par la présence 
d'une trentaine de nucléus laminaires et d'une vingtaine d'armatures (fig. 2). D'autre part, à cette série s'ajoutent deux concentrations collectées partiellement par C. Normand au début des années 1980 (fig. 2). Le débitage laminaire de belle facture de l'une lui valut la dénomination de "série à grandes lames ", les pièces à dos de l'autre, celle de "série à pièces à dos". À l'heure actuelle, la série Daguin actuellement conservée au musée d'Aquitaine à Bordeaux et la série à grandes lames sont toujours en grande partie inédites. La série à pièces à dos, quant à elle, a été partiellement présentée (Normand 1993 ; Simonet 2008).

\section{4 - Présentation de la série à pièces à dos}

4 Cette série a été découverte à environ 500 mètres à l'ouest de celle collectée par Emile Daguin (fig. 2). Elle a été recueillie dans l'urgence par C. Normand près du lieu-dit « Les Vignès » en juillet 1982. Pendant quatre jours, C. Normand a effectué une fouille de sauvetage en profitant du court délai imposé par la Société des Ciments de l'Adour qui exploitait alors la carrière. Environ 750 pièces furent collectées sur une surface de deux mètres carrés mais la dimension initiale de l'amas de taille demeure inconnue. Il s'agit donc de la collecte partielle et périphérique d'une concentration dont le cœur a été emporté par la décapeuse (Normand, com pers.). Son étude fut la problématique centrale de notre mémoire de maitrise (Simonet 2004).

\section{5 - Problématique paléo-sociologique}

$5 \quad$ L'originalité de cette série est de présenter un débitage maladroit auquel est associée une trentaine de pièces à dos de petites dimensions, inachevées et/ou ratées, dans un contexte d'atelier de taille pourtant paradoxalement propice au gaspillage. Conséquence directe de cette singularité, l'attribution gravettienne proposée par l'inventeur C. Normand est délicate à affiner (Normand 1993). Dans le cadre de cet article, c'est vers une réflexion paléo-sociologique que nous souhaiterions aboutir à l'aide d'une présentation précise des données. En effet, cette série ne pourrait-elle pas représenter l'un des plus anciens témoignages de l'apprentissage de la confection d'armatures lithiques au cœur du Paléolithique supérieur? Cette perspective de recherche s'avère d'autant plus passionnante qu'elle est archéologiquement très peu documentée et qu'aucun critère objectif permettant le diagnostic d'un stigmate d'apprentissage n'a pour l'instant été identifié (Pigeot 1988 ; Ploux 1991 ; Pelegrin 1995 ; Klaric 2006, en préparation). Des travaux fondateurs dans le domaine de la technologie lithique, il ressort que c'est davantage un faisceau d'indices auquel il est nécessaire de faire appel comme le permet le site d'Étiolles par exemple (Pigeot 1986, 1988, 1990 ; Pigeot dir. 2004; Ploux 1989, 1991; Karlin et al. 1993; Pelegrin 1995; Bodu 1994). Néanmoins, les différents niveaux de savoir-faire ou de compétence distingués dans ces études concernent le débitage laminaire. La documentation de l'apprentissage d'armatures lithiques au Paléolithique supérieur est, quant à elle, inédite et c'est en cela que Tercis offre un terrain d'étude innovant qui mérite, à l'avenir, d'être développé. 


\section{6 - Données stratigraphiques}

Les descriptions stratigraphiques des formations superficielles recouvrant les strates calcaires de l'anticlinal de Tercis renvoient à une échelle d'étude large, de l'ordre de plusieurs centaines de mètres carrés. Des variations locales sont donc envisageables.

7 Une couche de sable éolien correspondant au stade isotopique 2 a été constatée dans cette région (Thibault 1970) (fig. 3). À la base de cette couche de sable éolien, on observe sur l'ensemble du site de Tercis un niveau $2 b$ qui contient un mélange de plusieurs occupations (fig. 3), (Texier 1993). « Sur un peu plus de trois cents objets, il y a une quarantaine d'outils dont trois burins de Noailles, mais aussi des grattoirs carénés, des burins des Vachons et des racloirs. Il semble donc y avoir au moins trois industries représentées: Moustérien sensu lato, Aurignacien et Périgordien à burins de Noailles» (Normand 1993 p. 31).

8 La série présentée ici a été découverte dans ce dépôt de sables éoliens, à la base du niveau $2 a$ qui surmonte le niveau $2 b$ (fig. 3 ). Si le niveau $2 b$ offre, à l'échelle du site de Tercis, une association de pièces hétérogènes, les premières données typologiques et taphonomiques du niveau $2 \mathrm{a}$ semblent converger vers l'individualisation d'une concentration homogène. En effet, si les silex retrouvés dans le niveau sous-jacent possèdent une légère patine, ceux provenant du niveau $2 a$, attribuables au Gravettien, sont d'un aspect très frais.

En revanche, une industrie solutréenne se rencontre sous 20 centimètres de sables au lieu-dit Saussaye à quelques centaines de mètres de distance du lieu de découverte des séries gravettiennes. Or, l'occupation solutréenne est considérée comme postérieure à la mise en place des sables sous-jacents, de caractère éolien (Thibault 1970). Ces sables auraient donc été remaniés par endroits. Par conséquent, ils ne fixent pas un terminus ante quem certain. Néanmoins, $C$. Thibault propose le schéma suivant: le Solutréen se place au sommet des sables tandis que l'Aurignacien se situe à la base. Par corrélation et en ce qui concerne le niveau $2 a$, nous pouvons en déduire que nous sommes probablement en présence de Gravettien. 
Figure 2 - Localisation des principales concentrations gravettiennes de Tercis par rapport à la carrière et aux affleurements géologiques du Secondaire liés à l'anticlinal. A à $\mathrm{E}$ : paliers d'exploitation de la carrière. $N^{\circ} 1$ : série à grandes lames. $N^{\circ} 2$ : série à pièces à dos. $N^{\circ} 3:$ série Daguin. Dessin et photographie A. Simonet.

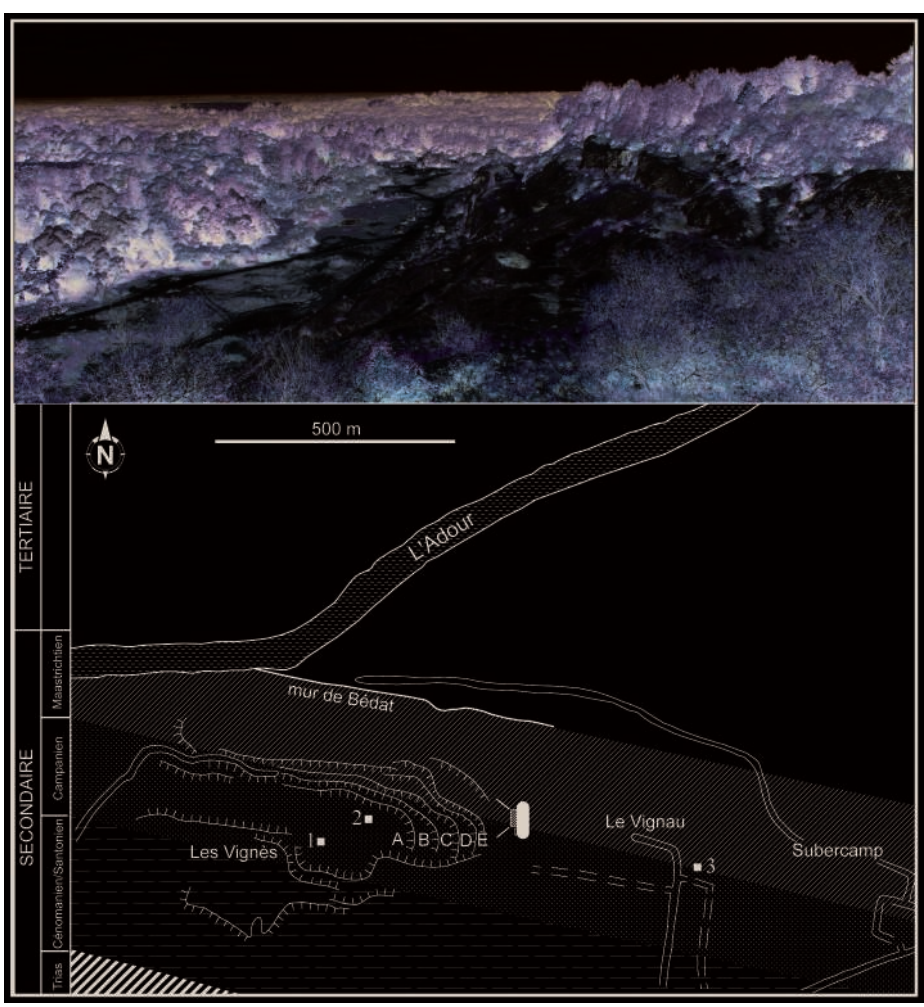

Figure 3 - Stratigraphie des couches supérieures des Vignès. D'après Texier 1993, fig. 2.

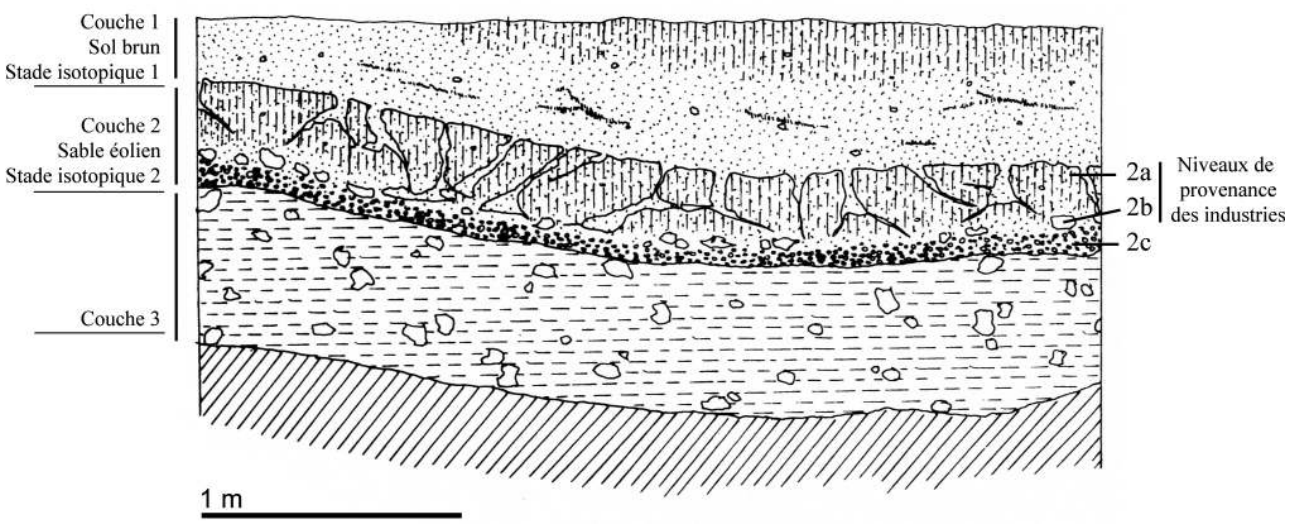

\section{2 - Présentation de la série à pièces à dos}

\section{1 - Présentation synthétique du corpus}

10 L'assemblage est dominé par les produits de débitage qui indiquent la recherche de petits produits laminaires. Les outils sont essentiellement représentés par les pièces à dos. La présence des outils domestiques est marginale avec quatre pièces à encoche et trois produits laminaires retouchés. Les produits de débitage sont essentiellement constitués d'éclats et d'esquilles avec 512 exemplaires. Ces derniers sont complétés par 
171 produits lamino-lamellaires, sept nucléus et cinq percuteurs durs aménagés sur des galets de quartzite d'origine locale (tabl. 1).

\section{2 - Les matières premières}

11 Le silex utilisé est quasi-exclusivement issu des formations de calcaire du Sénonien supérieur de la grande carrière de Tercis (tabl. 1). On y trouve en effet, dans un secteur très localisé, un silex de très bonne qualité : le «silex de type Tercis » qui se décline sous trois variétés. C'est surtout la première variété (a), un silex maastrichtien grisnoir, qui a été utilisée pendant la Préhistoire (fig. 2). La quasi-totalité des éléments de la série à pièces à dos est d'ailleurs confectionnée dans cette matière. C'est un silex gris à noir, translucide et de grain fin, montrant de rares recristallisations et des taches rouge-orangé caractéristiques. Sa marge de variabilité est importante : il peut ainsi se présenter sous une couleur gris/brun assez éloignée du noir initial. C'est un silex de très bonne qualité même s'il est souvent d'aspect un peu sec. Il se patine en gris-bleuté allant jusqu'au blanc profond. La longueur des rognons dépasse rarement 25 centimètres (Normand 2002).

12 La troisième variété (c) du silex de Tercis se retrouve également dans cette série (tabl. 1). Il s'agit d'un silex blanc-gris zoné qui n'a pour l'instant jamais été retrouvé en place dans les couches géologiques de la carrière de Tercis. On en retrouve dans des collectes de surface dans un rayon de deux/trois kilomètres (C. Normand, com. pers.). Son exploitation semble avoir été très faible. Son exportation est attestée dans des sites comme Brassempouy et Isturitz mais reste exceptionnelle. Il est caractérisé par un cortex fin, une zone sous-corticale de très bonne qualité et une diminution progressive de cette homogénéité sous-corticale à mesure que l'on s'approche du cœur grenu. Celui-ci est traversé de minces couches mieux silicifiées qui lui donnent son aspect zoné (Normand 2002). La série gravettienne à grandes lames exploite cette variété. Certaines lames de cette série dépassent ainsi 20 centimètres, ce qui est exceptionnel dans le contexte régional de la Chalosse. Dans la série à pièces à dos, seuls un nucléus et deux éclats associés présentent cette variété du silex de type Tercis. Le gîte n'ayant pas encore été découvert, la détermination précise de ce silex reste à faire. Quelques oursins caractéristiques du Sénonien ont néanmoins été identifiés (C. Normand, com. pers.).

13 Un nucléus et un produit laminaire se distinguent par un silex gris-blanc de bonne qualité, peut-être une sous-variété du silex maastrichtien gris-noir de type Tercis (C. Normand, com. pers.). Cette matière détonne au sein de la majorité des pièces en silex maastrichtien gris-noir, de qualité médiocre (tabl. 1).

14 Enfin, deux éclats ont été confectionnés dans du silex du type Flysch des calcaires de Bidache (tabl. 1). Ce silex présente une zone d'affleurement potentielle assez vaste dans la basse vallée de l'Adour. Les affleurements les plus proches de Tercis sont localisés à une vingtaine de kilomètres au sud. Il s'agit notamment de la matière première siliceuse la plus utilisée par les Gravettiens de la grotte d'Isturitz (Simonet 2010). Cette variété s'observe fréquemment sous forme de plaquettes dont l'épaisseur est le plus souvent inférieure à dix centimètres. Il s'agit d'un silex de grain moyennement fin, souvent opaque et de couleur grise, avec de fréquents litages parallèles à l'axe des plaquettes et signalés par une multitude de micro-ponctuations (Normand 2002). 
Tableau 1 - Décompte de la série à pièces à dos et distribution des matières premières.

\begin{tabular}{|c|c|c|c|c|c|c|c|}
\hline & \multirow[t]{2}{*}{ Quartzite } & \multirow{2}{*}{$\begin{array}{l}\text { Silex type } \\
\text { "noir de } \\
\text { Tercis" }\end{array}$} & \multirow{2}{*}{$\begin{array}{l}\text { Silex type } \\
\text { "gris zoné } \\
\text { de Tercis" }\end{array}$} & \multirow{2}{*}{$\begin{array}{r}\text { Silex gris- } \\
\text { blanc } \\
\text { type } \\
\text { "Tercis" ? }\end{array}$} & \multirow{2}{*}{$\begin{array}{l}\text { Silex type } \\
\text { "Flysch" }\end{array}$} & \multirow{2}{*}{\multicolumn{2}{|c|}{ Total }} \\
\hline & & & & & & & \\
\hline & & $\mathbf{N}$ & $\mathbf{N}$ & $\mathbf{N}$ & $\mathrm{N}$ & $\mathbf{N}$ & $\%$ \\
\hline Éclat et fragment & & 508 & 2 & & 2 & 512 & 69 \\
\hline Produit lamino-lamellaire & & 170 & & 1 & & 171 & 23,1 \\
\hline Chute de burin & & 1 & & & & 1 & 0,1 \\
\hline Nucléus & & 5 & 1 & 1 & & 7 & 1 \\
\hline Casson & & 1 & & & & 1 & 0,1 \\
\hline Percuteur & 5 & & & & & 5 & 0,7 \\
\hline Débris & & 2 & & & & 2 & 0,3 \\
\hline Total débitage & & 687 & & & & 699 & 95 \\
\hline Pièce à encoche & & 4 & & & & 4 & 0,5 \\
\hline Produit laminaire retouché & & 3 & & & & 3 & 0,4 \\
\hline Total outils "domestiques" & & 7 & & & & 7 & 0,9 \\
\hline Pièce à dos diverse & & 26 & & & & 26 & 3,5 \\
\hline Lamelle à dos & & 6 & & & & 6 & 0,8 \\
\hline Total armatures & & 32 & & & & 32 & 4,3 \\
\hline Total & 5 & 726 & 3 & 2 & 2 & 738 & $100 \%$ \\
\hline
\end{tabular}

\section{3 - Les armatures}

La quasi-totalité des 34 pièces à dos révèle un faible niveau d'investissement technique (fig. 4 et 5). D'une manière générale, le dénominateur commun entre ces pièces est leur aspect « atypique ». Elles sont caractérisées par l'absence d'une sélection normative des supports, en décalage avec le comportement gravettien classique qui consiste justement à valoriser la recherche de supports rectilignes et réguliers pour les armatures lithiques avec une corrélation fréquente entre l'orientation de la pointe et l'axe de débitage du support, comme le montrent par exemple les pointes à dos gravettiennes de la grotte d'Isturitz (Simonet 2010).

16 Par ailleurs, leur retouche est dans la grande majorité des cas incomplète. Ainsi, ces pièces en cours de fabrication sont particulières en ce qu'elles ne semblent pas fonctionnelles. C'est pourquoi l'idée selon laquelle ces pièces pourraient être la conséquence d'un exercice d'apprentissage a été émise par C. Normand, dès leur découverte au début des années 1980 (Normand 1993). Seules trois lamelles à dos se distinguent par la qualité du support, la régularité de leur retouche ainsi que par leur dimension (fig. 6 - nos 1 à 3 ).

17 Inversement, les autres pièces à dos ont en commun d'être imparfaites ou inachevées. L'hétérogénéité des supports sélectionnés est particulièrement frappante. Des lames de flanc ont ainsi été choisies (fig. $4-\mathrm{n}^{\circ} 1$ et fig. 5 - nos $4,5,8,9$ ), des éclats laminaires (fig. 4 - nos 7 et 9 et fig. 5 - nos $1,3,7,12,13$ ), une néo-crête partielle distale (fig. 4 - $\mathrm{n}^{\circ} 8$ ), un éclat lamellaire (fig. $5-\mathrm{n}^{\circ}$ 6), des produits de plein débitage dont la régularité ou la rectitude sont mauvaises et/ou présentent une forte ondulation (fig. 5 - nos 2, 10, 11). En définitive, cette diversité des supports est la conséquence d'un choix économique puisque ce sont des déchets de taille qui ont été utilisés.

18 Excepté les trois lamelles à dos dont les supports sont à la fois rectilignes avec des bords et des nervures régulières et parallèles (fig. 6 - nos 1 à 3), seules quatre pièces ont 
été confectionnées sur des supports de qualité relativement bonne (fig. 4 - nos 2, 4, 5, 6). Parmi celles-ci, deux pièces posent le problème du lien entre la cassure et le façonnage du dos (fig. 4 - nos 4 et 6). Ces fractures pourraient avoir eu lieu lors du débitage, antérieurement à la confection du dos. Ce seraient ainsi des fragments de produits de plein débitage qui auraient été choisis et non des produits laminaires bruts entiers. Le support d'une pièce, bien que régulier, est de très faible dimension, en décalage par rapport au gabarit moyen des produits débités (fig. $4-n^{\circ}$ 5). Finalement, une seule pièce possède un gabarit assez important avec une épaisseur de près de $5 \mathrm{~mm}$ et une largeur d'environ $8 \mathrm{~mm}$ tout en étant assez rectiligne et régulière (fig. $4-\mathrm{n}^{\circ} 2$ ). Néanmoins, elle n'a pas été aménagée sur un support de plein débitage puisque le négatif d'un enlèvement perpendiculaire à l'axe du débitage du support est présent sur la surface supérieure.

Si l'ensemble de ces supports se révèle inadapté à la fabrication de pièces à dos, ils dévoilent une expérience importante des principes de la taille du silex. Certains supports de pièces à dos témoignent ainsi de l'extraction de lames de flanc pour corriger le cintre et accentuer le phénomène de convergence de la partie distale de la table laminaire dans l'objectif de créer des produits de plein débitage naturellement effilés en partie distale (fig. 5 - nos 4 et 5). Sont également présents des enlèvements destinés à réaménager la table laminaire (fig. 4 - $\mathrm{n}^{\circ} 8$ et fig. 5 - nos 8 et 9), une néo-crête distale pour maintenir une convexité longitudinale (fig. $4-\mathrm{n}^{\circ} 8$ ) ainsi que le recours à un plan de frappe opposé comme l'illustrent des négatifs d'enlèvements opposés sur la surface supérieure de certaines pièces à dos (fig. $4-\mathrm{n}^{\circ} 2$ et fig. 5 - $\mathrm{n}^{\circ} 14$ ).

L'opposition entre la médiocrité des pièces à dos et la bonne connaissance des principes de la taille du silex représente le premier d'une série de contrastes qui traversent cet assemblage lithique. Les supports donnent l'impression d'avoir été sélectionnés parmi les plus mauvais produits issus d'un débitage laminaire exigeant un niveau de compétence élevé. Par conséquent, il peut sembler paradoxal que les personnes qui ont fabriqué les supports destinés à être transformés en pièces à dos d'une part, et celles qui ont aménagé les dos d'autre part, soient les mêmes.

Ces premières données concernant les armatures lithiques sont d'ores et déjà cohérentes avec l'hypothèse d'un exercice d'apprentis auxquels auraient été réservés des déchets de débitage. Cette hypothèse expliquerait également l'abandon de certaines pièces à dos non fragmentées (fig. 4 - nos 1,8 et 9) et la présence d'une pièce dont le tailleur savait pertinemment avant de débuter le façonnage qu'elle ne pourrait jamais être fonctionnelle (fig. $4-\mathrm{n}^{\circ}$ 8). Cette hypothèse expliquerait également la diversité des morphologies des pièces à dos : le geste importerait plus que l'objet fini.

Dans un contexte d'atelier de taille à proximité d'un gîte de matière première, la probabilité d'un gaspillage du silex sous forme de confection de pièces atypiques et/ou non-fonctionnelles est néanmoins plus élevée et incite à prendre avec précaution l'interprétation des pièces à dos comme vestiges de l'apprentissage de l'aménagement d'un dos. 
Figure 4 - Pièces à dos. Dessins A. Simonet.

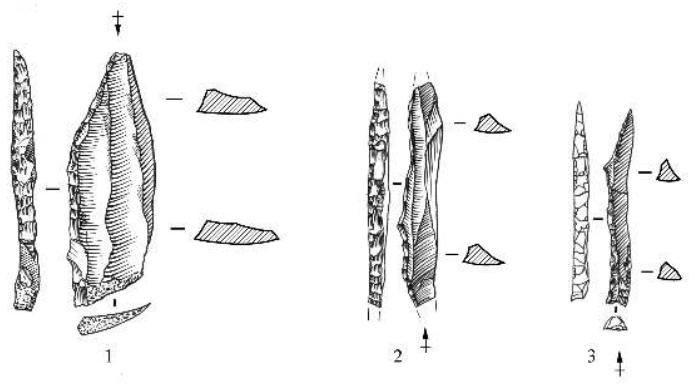

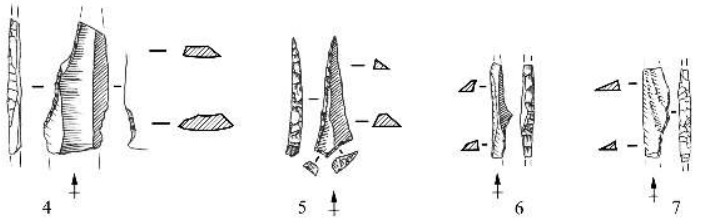

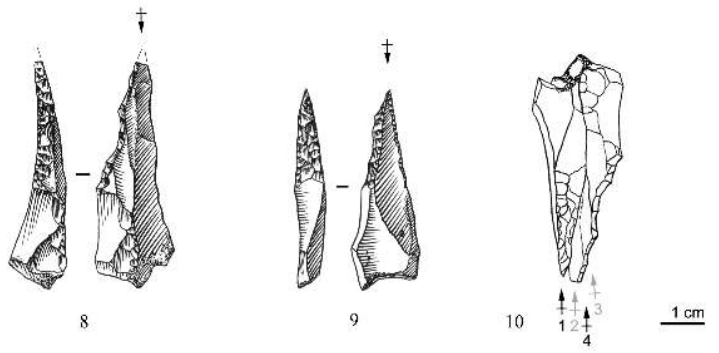

Figure 5 - Pièces à dos. Dessins A. Simonet.
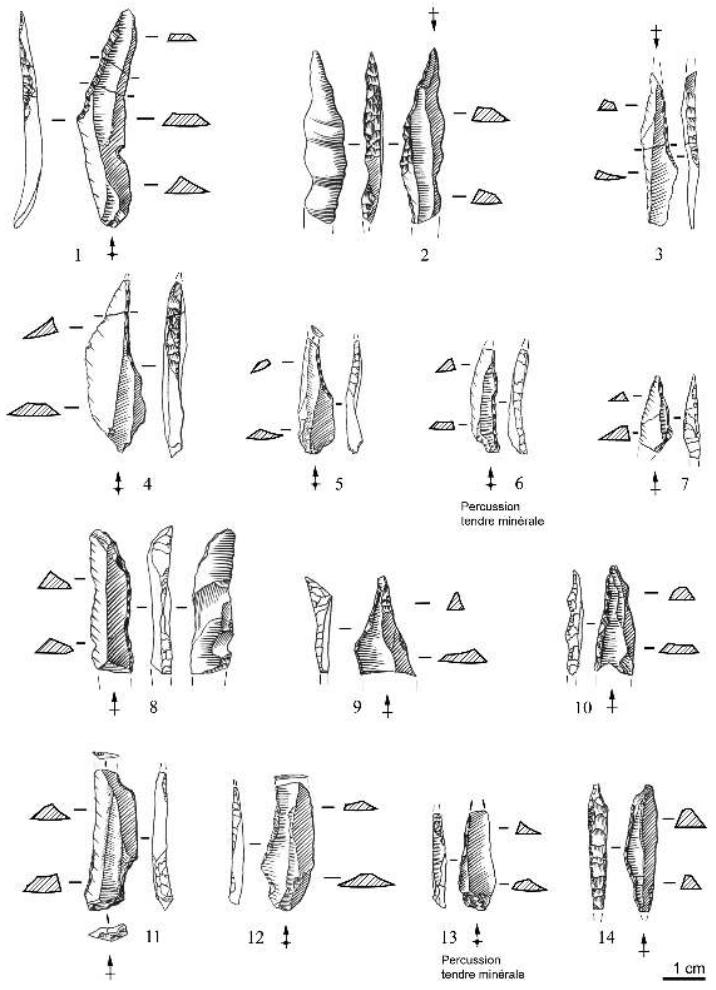
Figure 6 - Lamelles à dos et micro-pièces à dos. Dessins A. Simonet.
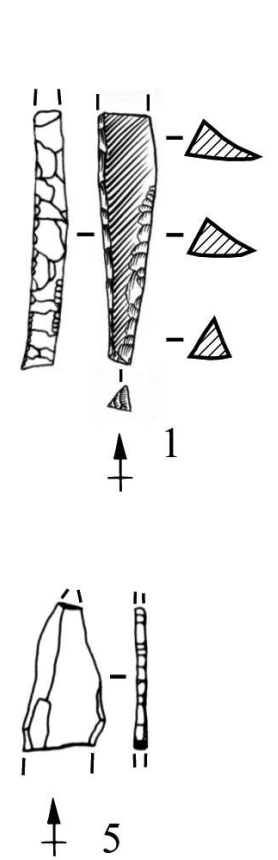

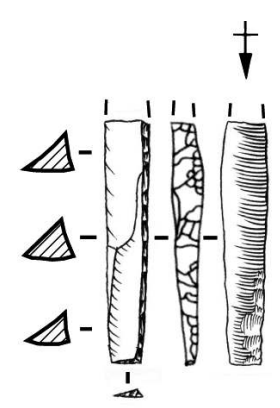

2

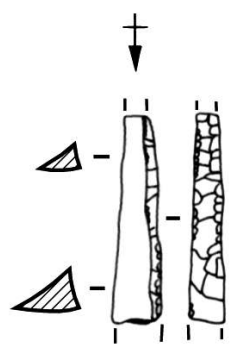

3

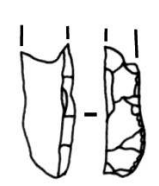

f 8
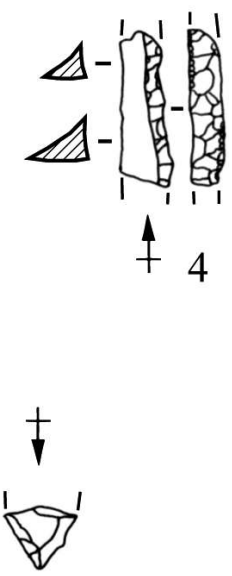

$9 \quad 1 \mathrm{~cm}$

\section{4 - Les nucléus}

Le nucléus $\mathrm{n}^{\circ} 1$ est un nucléus prismatique de belle facture possédant deux plans de frappe opposés et une table laminaire très cintrée d'une dizaine de centimètres de hauteur (fig. 7). Des négatifs d'enlèvements perpendiculaires à l'axe de la table laminaire suggèrent l'utilisation de crêtes antérieure et postérieure. C'est le seul nucléus confectionné dans un silex gris-blanc de bonne qualité, de type Tercis, mais dont l'identification précise est délicate. Bien qu'il ne remonte pas sur le nucléus, il est probable que le seul produit laminaire de matière première identique provienne de son exploitation (fig. 16 - nº 5). Il s'agit d'une lame sous-crête débitée à la percussion tendre minérale. Sa longueur importante la distingue clairement de l'ensemble des produits laminaires en silex gris-noir de Tercis, de gabarit plus petit comme nous le verrons par la suite.

Le nucléus $n^{\circ} 2$ possède une mauvaise carène ainsi qu'un mauvais cintrage (fig. 8). La table laminaire est trop plate et les enlèvements ont réfléchi. Le tailleur a mis en place une demi-crête latérale (fig. $8-n^{\circ} 4$ ) mais il n'a pas corrigé la convexité distale longitudinale de la table laminaire. De fait, l'enlèvement suivant a également réfléchi (fig. $8-n^{\circ}$ 5) et le nucléus a été abandonné. La présence de cette légère proéminence dans la partie distale de la table qui n'a pas été supprimée par le tailleur peut être interprétée comme une erreur de sa part.

Le nucléus $n^{\circ} 3$ investit un gros débris cortical (fig. 9). L'extraction de l'éclat qui a permis d'installer un plan de frappe a simultanément débarrassé le support de sa partie la plus impropre. Aucune mise en forme ni préparation du plan de frappe n'a été effectuée. Pourtant, l'angulation entre le plan de frappe et la table est beaucoup trop ouverte (proche de $90^{\circ}$ ) pour produire des enlèvements laminaires. Deux enlèvements laminaires ont néanmoins été extraits le long d'arêtes naturelles. Le premier 
enlèvement est probablement cortical. Le second enlèvement a rebroussé et le nucléus est abandonné sans avoir donné de produit exploitable.

Le nucléus $\mathrm{n}^{\circ} 4$ investit un petit éclat d'environ sept centimètres de hauteur (fig. 10). Après l'ouverture d'un plan de frappe et une légère préparation de la corniche par abrasion, un débitage de petits produits laminaires a débuté par le flanc droit et a envahi progressivement la tranche de l'éclat. Dans une première étape, quatre produits laminaires au moins (fig. $10-$ nos $3_{1}$ à $3_{4}$ ) ont été extraits selon un agencement quasiment semi-tournant qui aplatit la table laminaire. Le cintre devenu trop ouvert, les deux éclats laminaires débités ensuite à la percussion tendre minérale et remontés sur le nucléus, ont réfléchi (fig. 10 - nos $4_{1}$ et $4_{2}$ ). L'avant-dernier enlèvement laminaire, dont la partie distale brisée a été remontée sur le nucléus, a quant à lui outrepassé tandis que le dernier enlèvement effectué avant l'abandon du nucléus a réfléchi (fig. 10 $\left.-n^{\circ} 5\right)$.

Le nucléus $\mathrm{n}^{\circ} 5$ a été aménagé sur un petit bloc de silex roulé et gélifracté, de forme ovoïde et d'environ neuf centimètres de hauteur (fig. 11). Ce petit bloc a été fracturé en deux (fig. $11-\mathrm{n}^{\circ} 1$ ) puis l'un des fragments d'environ six centimètres de hauteur a été utilisé comme support de nucléus. Deux enlèvements laminaires en partie corticaux ont été détachés (fig. 11 - nos $2_{1}$ et $2_{2}$ ). Ont suivi l'extraction d'un produit laminaire souscortical (fig. $11-n^{\circ} 3$ ) et celle d'un dernier produit laminaire réfléchi qui ont été remontés (fig. $\left.11-n^{\circ} 4\right)$. Son talon présente les traces d'une légère préparation par abrasion.

L'exemplaire $\mathrm{n}^{\circ} 6$ est un nucléus prismatique à deux plans de frappe opposés, d'une hauteur de dix centimètres environ (fig. 12 et 13). Il a probablement été aménagé sur un petit bloc. Plusieurs produits laminaires ont été remontés par $\mathrm{C}$. Normand et $\mathrm{S}$. Dartiguepeyrou. Ils semblent avoir été débités à la percussion tendre minérale. Ce nucléus a subi une mise en forme élaborée par rapport aux autres exemplaires avec la mise en place probable d'une crête postérieure (fig. $12-n^{\circ} 1$ ) et la mise en place certaine d'une crête antérieure à deux versants (fig. $12-\mathrm{n}^{\circ} 2$ ). Le débitage est frontal et a permis l'extraction de produits laminaires de huit centimètres de longueur environ. Une dizaine de produits laminaires a pu être extraite avant les derniers exemplaires remontés sur le nucléus (fig. 12 - nos 3 et 4). Dans l'ordre chronologique du débitage, le premier produit laminaire remonté est une lame sous-crête (fig. 12 - nº 5). À l'instar du nucléus $\mathrm{n}^{\circ} 2$, une proéminence distale de la table laminaire a gêné le bon détachement des produits laminaires suivants qui ont réfléchi (fig. 13 - nos $6_{1}$ à $6_{3}$ ). Le tailleur a tardivement tenté, par l'extraction du produit laminaire $6_{4}$, de corriger ces réfléchissements à l'aide d'une néo-crête partielle. Par ailleurs, l'hétérogénéité du silex a induit de fortes ondulations qui ont compliqué le débitage. Se trouvant face à un silex de mauvaise qualité, le tailleur ne pouvait guère améliorer l'état de la table. Il a alors décidé d'investir le flanc droit (fig. $13-\mathrm{n}^{\circ}$ 8) après une légère correction de la carène à partir du plan de frappe $n^{\circ} 2$ (fig. $13-n^{\circ} 7$ ). Les quatre produits laminaires alors extraits à partir du plan de frappe $\mathrm{n}^{\circ} 1$ ont réfléchi malgré la mise en place d'une néo-crête distale partielle, probablement entre les enlèvements $8_{3}$ et $8_{4}$. Toujours à partir du plan de frappe $n^{\circ} 1$, le tailleur est alors revenu dans l'axe initial frontal du débitage afin d'extraire les produits laminaires $9_{1}$ à $10_{2}$. Seul l'enlèvement $9_{2}$ s'avère manquant. Les deux derniers enlèvements $10_{1}$ et $10_{2}$ ont réfléchi (fig. 13). Le nucléus est finalement abandonné avec une table très accidentée. 

nucléus $\left.n^{\circ} 3,5,6,7\right)$. Dans un contexte où les blocs de bonne qualité sont nombreux, eu égard aux nombreux affleurements de silex disponibles et au nombre important d'objets récoltés sur le site depuis le XIXè siècle, il est particulièrement étrange que le(s) tailleur(s) n'ai(en)t sélectionné que des blocs de silex de petite dimension, gélifractés, roulés ou altérés. Leur exploitation ne peut certainement pas être interprétée comme une difficulté d'approvisionnement en bonne matière première. $\mathrm{Et}$ lorsque le silex est de meilleure qualité (nucléus $n^{\circ} 3$ et 4 ), les supports utilisés pour les nucléus sont de trop petites dimensions dans l'objectif de fabriquer une pointe à dos. Les quelques supports débités, quant à eux, n'ont pas de vocation économique puisque la plupart ont pu être remontés sur les nucléus. Ces débitages ne possèdent donc aucun potentiel économique et les supports de nucléus exploités donnent justement l'impression d'avoir été sélectionnés pour leur caractère improductif. Seul le nucléus $\mathrm{n}^{\circ} 1$ a été productif. Or, c'est également le seul nucléus dont les produits n’ont pas été retrouvés.

Concernant les modalités opératoires, seul le nucléus $n^{\circ} 1$ se distingue par une gestion complexe du débitage et n'a clairement pu être exploité par un tailleur apprenti (fig. 7). Les nucléus nos 8 et 9 ont également pu être exploités par une ou plusieurs personnes 
compétentes au vu d'opérations de cintrage et de carénage selon des procédés diversifiés : néo-crête médiane distale, néo-crête latérale, second plan de frappe de réfection, rythme de débitage faisant alterner les enlèvements de chaque côté d'un axe médian de manière à conserver un bon cintre (fig. 15). Le nucléus $\mathrm{n}^{\circ} 6$ est un cas équivoque (fig. 12 et 13). L'ensemble des procédés techniques sont assimilés (cintrage, crête latérale, second plan de frappe de réfection). L'irrégularité et la mauvaise rectitude des supports peuvent tout aussi bien être interprétées comme les conséquences d'une matière première médiocre que comme une maladresse du tailleur. L'utilisation trop tardive du second plan de frappe pour corriger la carène est la seule erreur envisageable. La sélection d'une matière médiocre, qui peut généralement être interprétée comme un des critères de distinction d'un débitage d'apprenti, est ici particulièrement problématique. De manière paradoxale, un silex de bonne qualité dévoile les maladresses d'un tailleur sans avoir à faire intervenir le problème de l'adaptation et de la contrainte de la matière. Demeurent les nucléus nos 2, 3, 4, 5 et 7 qui ne subissent aucune mise en forme et qui n'ont produit chacun que quelques supports médiocres de petite dimension (fig. 8, 9, 10,11,14). Le nucléus $n^{\circ} 5$ est sans doute le cas le plus intéressant dans l'argumentation d'un débitage d'apprenti : la qualité du silex n'est pas adaptée à un débitage laminaire et le tailleur savait pertinemment qu'aucun support utilisable ne pourrait être produit (fig. 11).

Figure 7 - Nucléus $n^{\circ} 1$ avec une table laminaire cintrée et deux plans de frappe. Dessin A. Simonet.

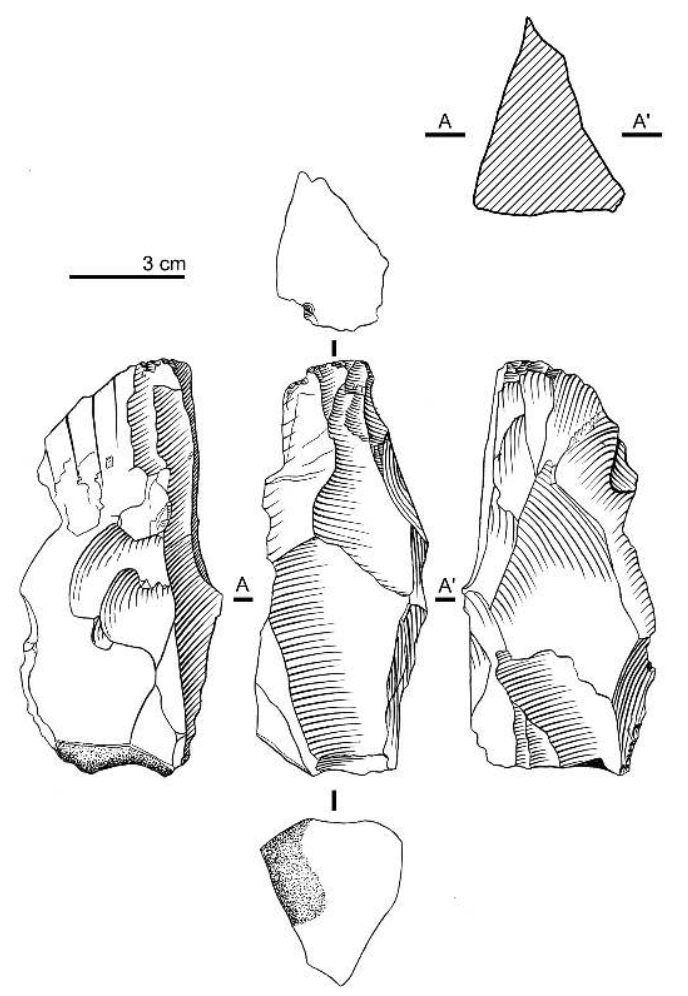


Figure 8 - Nucléus $n^{\circ} 2$ avec 5 produits remontés. Dessins A. Simonet.
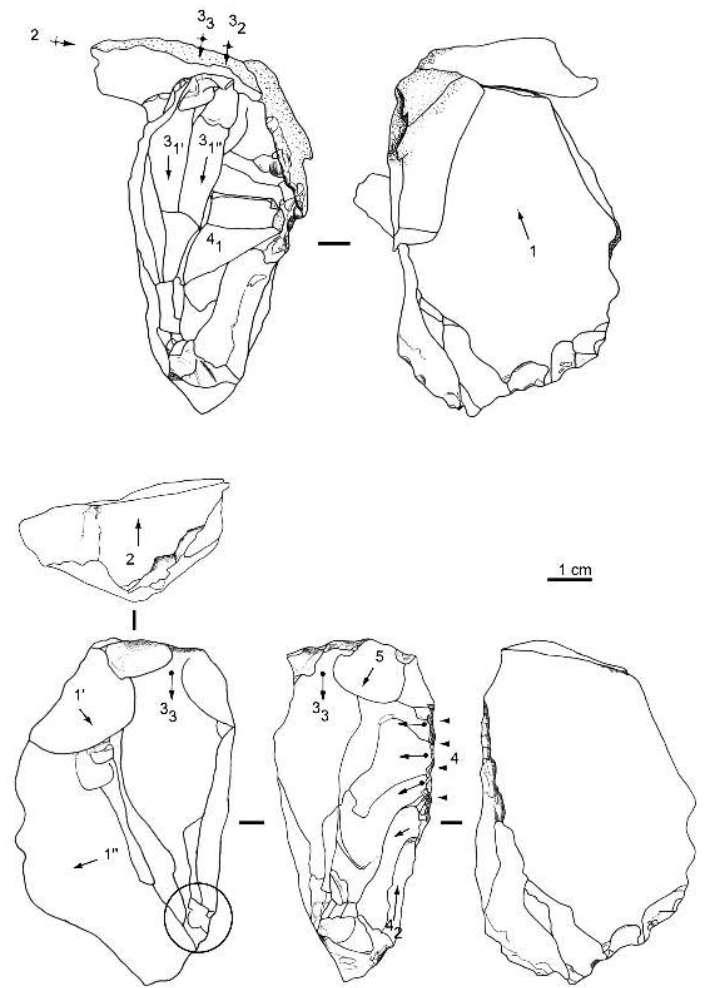

Figure 9 - Nucléus $n^{\circ} 3$ avec 2 produits remontés. Dessin A. Simonet.
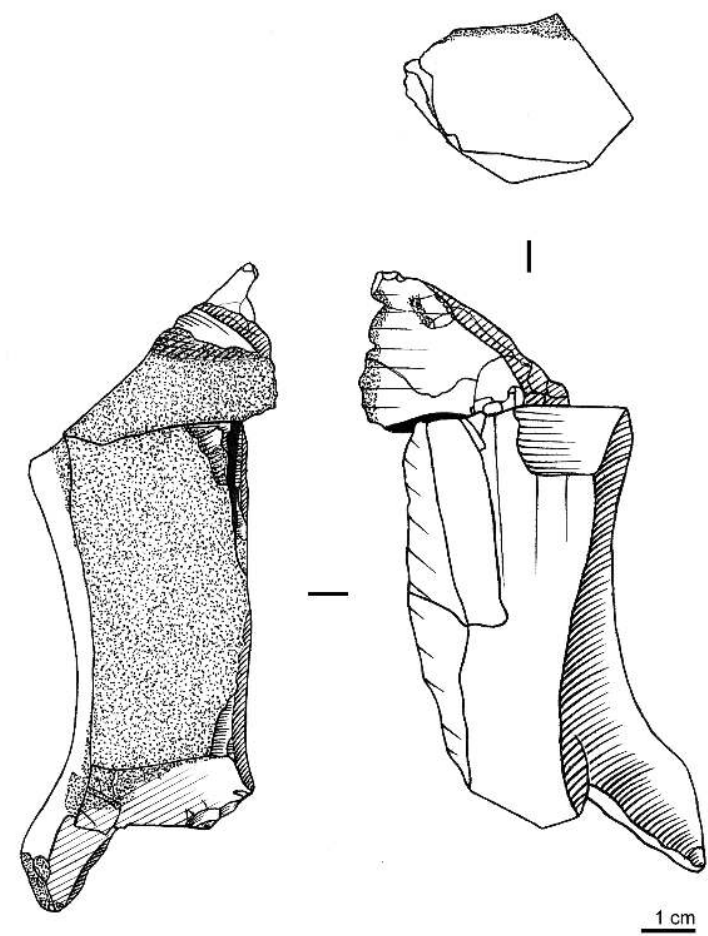


\section{5 - Les produits bruts de débitage}

En retirant du décompte les enlèvements remontés sur les nucléus, il reste 111 produits laminaires entiers et fragmentés. Parmi ces produits, 37 exemplaires sont entiers, soit exactement le tiers. Dix-huit raccords abaissent le nombre de pièces à 102 exemplaires (tabl. 2). D'une manière générale, ces produits laminaires sont peu ou pas patinés mais huit pièces portent une patine importante et 18 une légère patine. Toutes les pièces sont en silex noir maastrichtien de Tercis, excepté un grand produit laminaire en silex gris zoné de Tercis (fig. $16-\mathrm{n}^{\circ}$ 5). Parmi les 62 pièces entières ou brisées évoquant les produits recherchés lors du plein débitage, 17 sont entières (fig. 16 - nos 1 à 4). La majorité des produits est très rectiligne mais la régularité des bords et des nervures, présentes sur les surfaces supérieures, demeure moyenne. Seuls quatre produits laminaires entiers et de bonne facture peuvent donner une image correcte du gabarit recherché (fig. 16 - nos 1 à 4).

Les longueurs des produits laminaires entiers se concentrent entre cinq et six centimètres (fig. 17). En écartant les produits de réfection (lames à crête, lames de flanc, enlèvements de table) afin de mieux cibler les caractères métriques recherchés, les 62 produits laminaires et fragments de produits laminaires pris en compte présentent une largeur comprise entre 15 et $20 \mathrm{~mm}$ pour une épaisseur comprise entre 4 et $6 \mathrm{~mm}$ (fig. 18). Seule la lame en silex gris zoné se démarque nettement au sein de la série par un gabarit exceptionnel, avec une longueur de 12 centimètres (fig. $16-\mathrm{n}^{\circ} 5$ ).

Des produits de réfection, comme 12 produits de flanc à la fois courbes et torses avec des talons souvent obliques, une quinzaine de néo-crêtes latérales et médianes et trois enlèvements de table légèrement outrepassés, montrent l'utilisation de procédés assez variés pour conserver une table laminaire à la fois cintrée et rectiligne (fig. 16 - nos 6 et 7). S'y ajoute une quinzaine de produits laminaires portant des négatifs d'enlèvements opposés évoquant ainsi l'utilisation d'un second plan de frappe (fig. $16-\mathrm{n}^{\circ} 6$ ). De fait, l'ensemble des produits laminaires converge vers l'identification d'une chaîne opératoire unique dédiée à la confection de petits supports laminaires rectilignes et réguliers à partir de nucléus très cintrés unipolaires ou bipolaires.

Sur 67 produits laminaires dont le talon permet l'étude du mode de percussion, 44 produits laminaires portent des stigmates nets d'une percussion tendre minérale comme l'attestent la forte abrasion des talons, la présence d'un point d'impact souvent punctiforme ou réduit, l'esquillement particulier du bulbe et la présence fréquente à sa surface de rides fines et serrées (fig. 19) (Pelegrin 2000). L'abrasion de la corniche de ces produits laminaires est plus insistante que celle des produits remontés sur les nucléus. 
Figure 10 - Nucléus $n^{\circ} 4$ avec 3 produits remontés. Dessin A. Simonet.

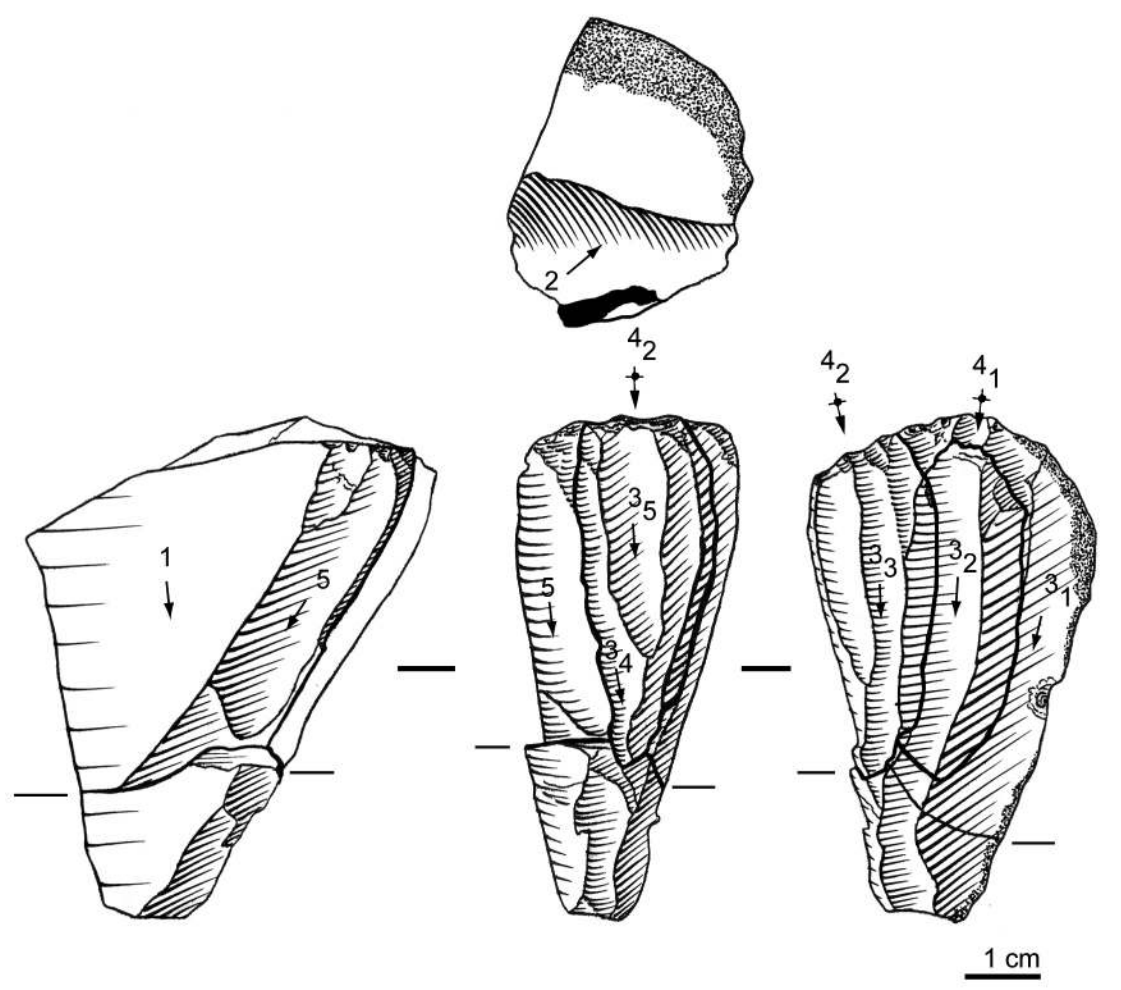

Figure 11 - Nucléus $n^{\circ} 5$ avec 3 produits remontés. Dessins A. Simonet.
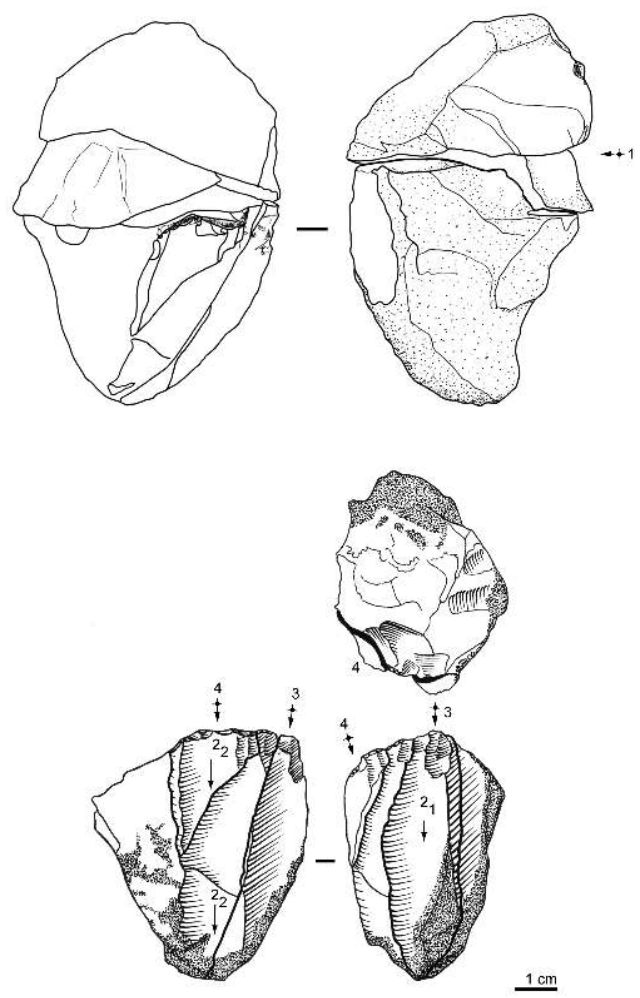
Figure 12 - Nucléus $n^{\circ} 6$ avec 15 produits remontés. Dessin A. Simonet.
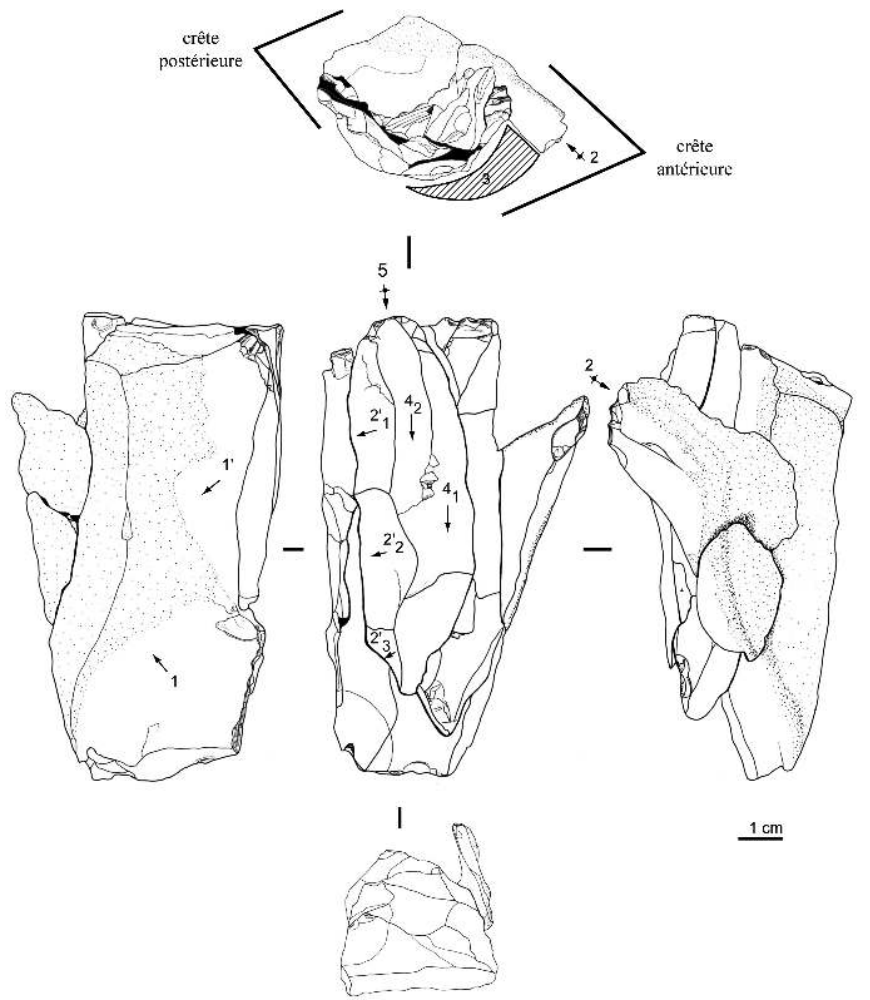

Figure 13 - Démontage progressif du nucléus n 6 . Dessins A. Simonet.

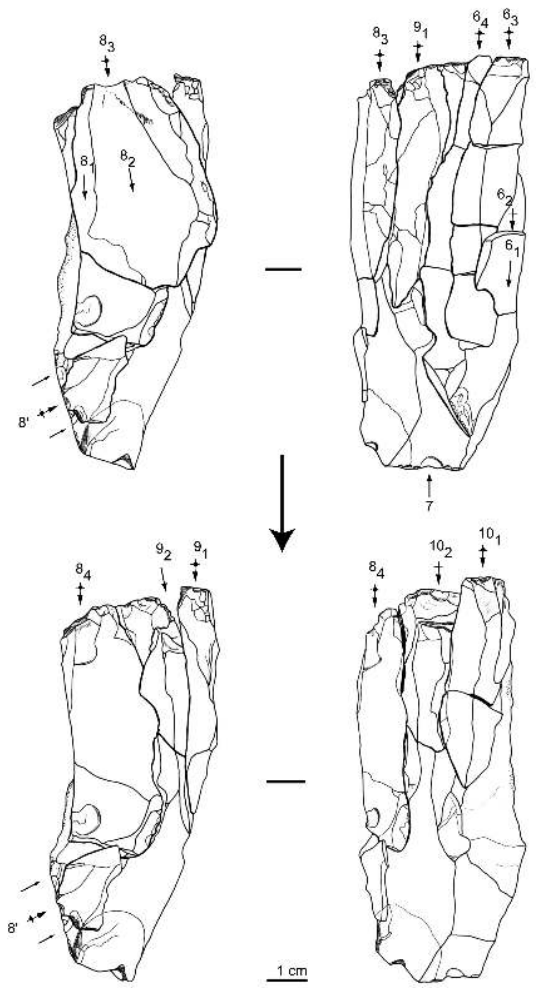


Figure 14 - Nucléus $n^{\circ} 7$ avec 2 produits remontés. Dessin A. Simonet.
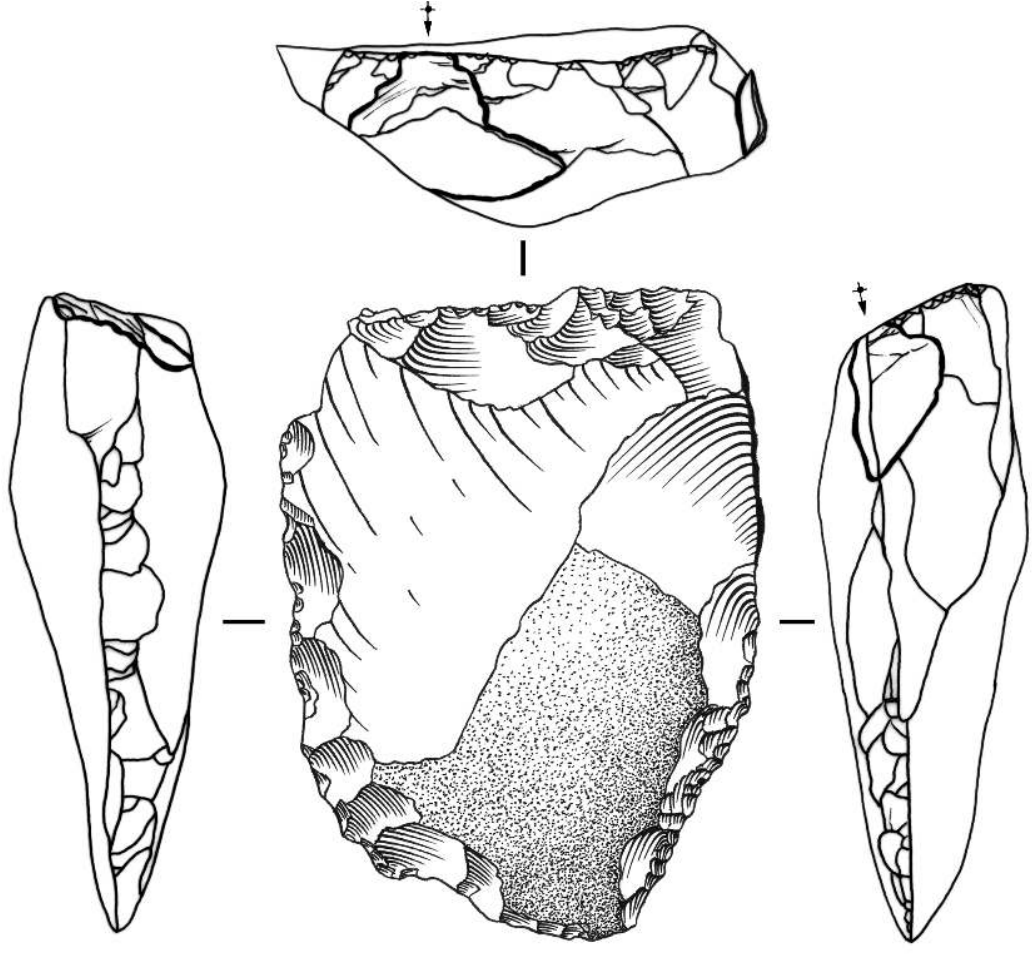

$1 \mathrm{~cm}$

Figure 15 - Remontage de 40 produits bruts. Photographie A. Simonet.

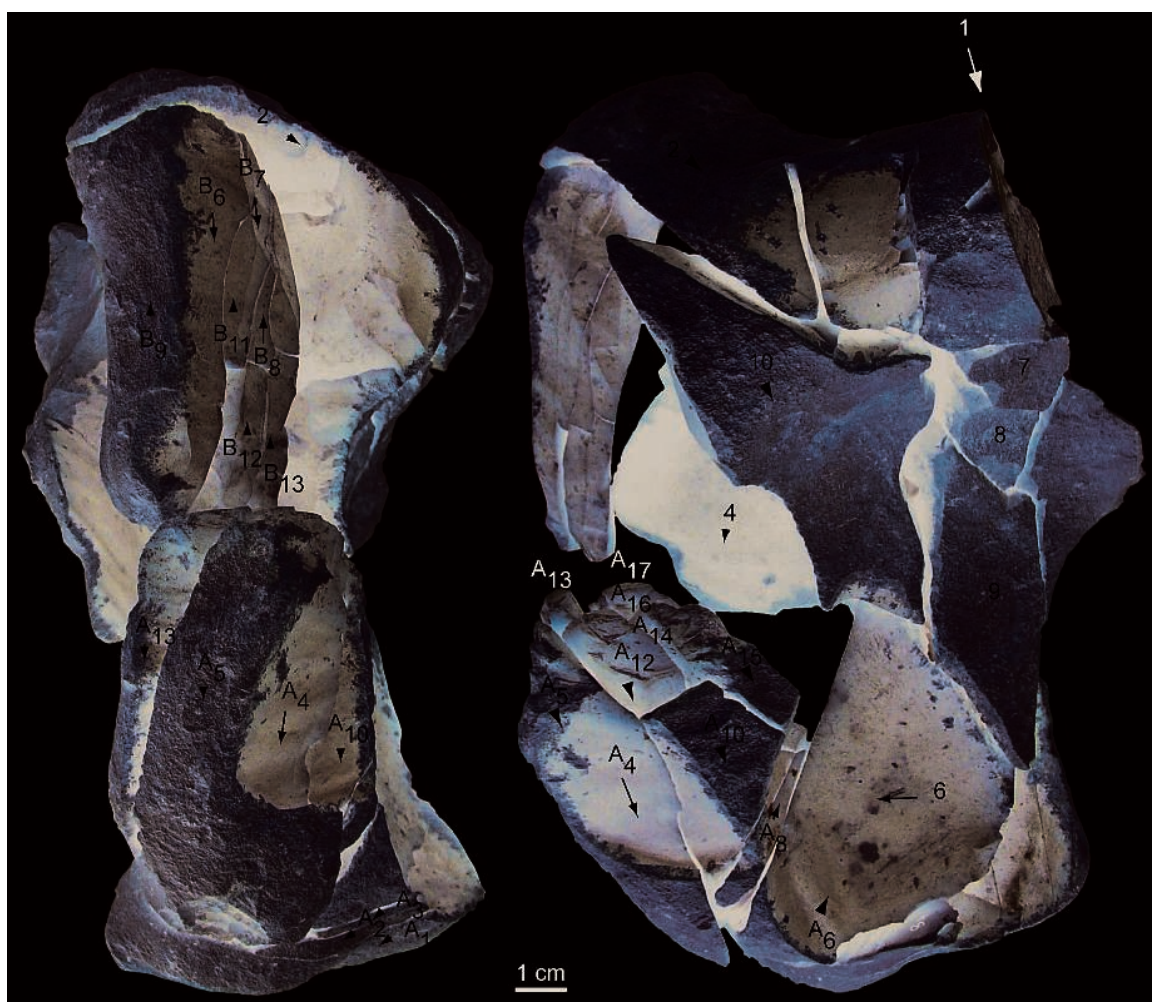




\section{3 - Un exemple d'apprentissage d'armatures à dos ?}

\section{1 - Les contradictions de la chaîne opératoire}

37 Le premier contraste de cette série est l'exploitation dominante d'une matière première médiocre, roulée et/ou gélifractée, dans un des meilleurs contextes gitologiques de la Chalosse.

38 Par ailleurs, deux types d'exploitation peuvent d'ores et déjà être remarqués : une exploitation sur des blocs de bonne qualité ayant fourni des supports de bonne facture comme le montrent les nucléus nos 1,8 et 9 ainsi qu'une grande partie des produits laminaires bruts (fig. 7, 15 et 16). Inversement, les autres nucléus et les produits qui y remontent témoignent de l'existence complémentaire d'une exploitation sur place de blocs ou d'éclats de qualité médiocre dont la plupart des éléments de la chaîne opératoire ont été retrouvés, et ont fourni des produits plus proches d'éclats laminaires dont le détachement est moins préparé (fig. 8 à 14). La différence entre les six nucléus ayant autorisé des remontages et les produits laminaires réguliers bruts débités dans du silex de bonne qualité est incontestable, surtout si l'on prend en considération le fait que ces produits abandonnés sont ceux de moindre qualité.

39 Cette production médiocre se retrouve au sein des supports transformés en pièces à dos (fig. 4 et 5). Les supports les plus mauvais ont été systématiquement sélectionnés tandis que certains supports laminaires de meilleure qualité n'ont pas été investis (fig. 16).

40 Le seul nucléus qui a été productif et qui possède des modalités opératoires complexes, en l'occurrence le nucléus $\mathrm{n}^{\circ} 1$, est, d'une part, le seul qui ait été confectionné dans un silex gris-blanc de bonne qualité et, d'autre part, le seul dont les produits issus de son exploitation n'ont pas été retrouvés (fig. 7). Seule une lame provenant probablement de son exploitation a été collectée (fig. $16-\mathrm{n}^{\circ}$ 5). Elle est curieusement de gabarit et de facture exceptionnels. Pour quelle raison ce nucléus et cette lame, probablement exploités dans une autre partie du site de Tercis ou dans une zone détruite de cette même concentration, se retrouvent-ils isolés?

41 Inversement, pourquoi les deux nucléus nos 8 et 9 (fig. 15) issus de l'exploitation d'un même bloc de silex noir de Tercis ne sont-ils pas associés aux produits de débitage? Ont-ils été exportés? Sont également absents les nucléus dont sont issus les produits laminaires bruts de qualité relativement bonne (fig. 16).

Cette série associe des pièces à dos à plusieurs nucléus dont la majorité des produits ont été remontés. Par conséquent, on peut affirmer que les pièces à dos n'ont pas été confectionnées à partir de supports issus de l'exploitation des nucléus. Au contraire, étant donné que les supports des pièces à dos sont des déchets de taille issus d'un débitage laminaire complexe, tout concourt à montrer qu'elles ont été aménagées sur des supports issus de nucléus productifs qui ne sont pas présents dans cette partie de la concentration.

43 Enfin, ces pièces à dos sont médiocres à l'instar des nucléus dont les produits ont été en grande partie remontés, contrastant ainsi avec la qualité de certains produits laminaires bruts et la grande compétence nécessaire à leur production et à l'exploitation du nucléus no 1 et des exemplaires absents. Quelles raisons ont poussé les tailleurs à confectionner des pièces à dos alors qu'ils savaient, avant de débuter la 
confection du dos, que certaines au moins ne pourraient pas être utilisées ? La même question se pose d'ailleurs pour la plupart des nucléus.

Tableau 2 - Décompte des produits laminaires bruts (hors produits remontés).

\begin{tabular}{|l|c|}
\cline { 2 - 2 } \multicolumn{1}{c|}{} & $\mathbf{N}$ \\
\hline Lame à pan cortical & 2 \\
\hline Lame sous-crête & 4 \\
\hline Lame de plein débitage & 62 \\
\hline Lame de recintrage & 12 \\
\hline Enlèvement de table & 3 \\
\hline Nèo-crête mèdiane & 13 \\
\hline Nèo-crête latérale & 2 \\
\hline Ėclat laminaire imise en fome ou entretien) & 4 \\
\hline Total & 102 \\
\hline
\end{tabular}

\section{2 - L'hypothèse de l'apprentissage}

Pris séparément, aucun des indices exposés précédemment ne représente un critère objectif de l'apprentissage de la taille du silex. Des causes différentes peuvent avoir les mêmes conséquences: un besoin rapide ne nécessitant pas une mise en forme complexe associée à une mauvaise matière première, des tests réalisés par un tailleur expérimenté pour s'échauffer avant de s'attaquer à des débitages exploitant une matière première de qualité, ou bien les conséquences d'un simple jeu... Néanmoins, le faisceau d'indices présents dans cet assemblage permet quant à lui de proposer une argumentation solide sur l'hypothèse d'un atelier d'apprentissage d'armatures lithiques à dos (fig. 20) :

- Les données pétrographiques : des tailleurs compétents ont-ils sélectionné des blocs de silex médiocre et des déchets de taille pour que les apprentis s'entraînent à la fois au débitage de lames rectilignes et à la confection de pièces à dos sans gaspiller le silex de bonne qualité ?

- Les données économiques : seul un nucléus a été productif. Cinq nucléus n'ont fourni, en moyenne, que deux ou trois produits laminaires irréguliers tandis que trois nucléus ont offert plus d'une dizaine de produits laminaires quasiment tous irréguliers. La plupart de ces produits laminaires n'ont pas été utilisés.

- Les données technologiques : ces nucléus reproduisent en plus maladroit et en plus petit, en l'adaptant à la médiocrité de la matière première, le concept gravettien de débitage basé sur une exploitation à deux plans de frappe hiérarchisés et à table laminaire cintrée destinée à une production de supports rectilignes. L'étude des produits laminaires bruts montre que le type recherché est unique. Il s'agit de lames rectilignes très normées d'environ $60 \mathrm{~mm}$ de longueur, de 15 à $20 \mathrm{~mm}$ de largeur et de $5 \mathrm{~mm}$ d'épaisseur. Le gabarit correspond exactement à celui nécessaire pour la confection d'une pointe à dos. En revanche, les modalités sont simplifiées et les procédés d'entretien et de réfection de la table laminaire ne sont pas toujours adaptés aux circonstances. Ces débitages représentent des versions médiocres de ceux présents dans les grands sites gravettiens voisins de Brassempouy et d'Isturitz.

- Les données spatiales: une exploitation de qualité a été effectuée au sein d'une concentration adjacente ou dans une zone différente de cette même concentration qui n'a malheureusement pas pu être collectée. Étant donné que ces pièces ont été retrouvées dans la zone périphérique de l'atelier de taille, il est possible qu'un débitage plus soigné ait eu lieu 
au cœur même de la concentration. Le nucléus $n^{\circ} 1$ et sa lame, ainsi que l'ensemble des produits laminaires de bonne qualité qui n'ont pas pu être débités sur les nucléus présents dans la série, en seraient les seuls témoins. Nous aurions ici de maigres indices d'une répartition spatiale différentielle des activités selon le degré de compétence.

- Les données " pédagogiques " : le nucléus $\mathrm{n}^{\circ} 1$ et son unique lame pourrait détenir le rôle de modèle pour guider les apprentis. Ces objets représenteraient l'exemple à suivre.

Figure 16 - Produits laminaires bruts. Certains exemplaires, de meilleure facture que les supports utilisés pour les pièces à dos, n'ont pourtant pas été retenus. Dessins A. Simonet.
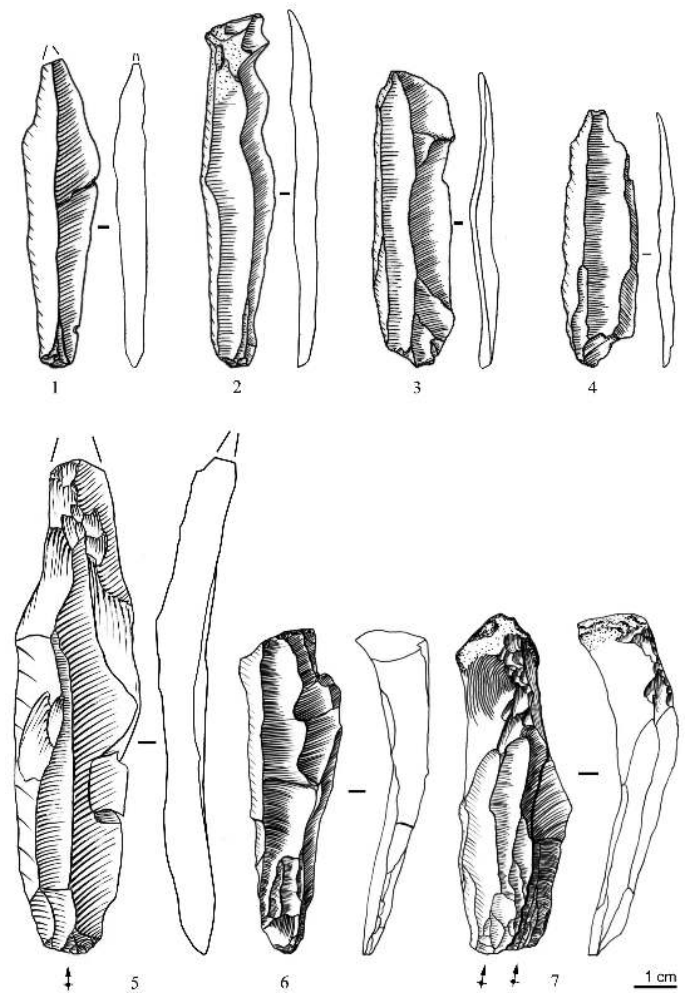

Figure 17 - Longueur des produits laminaires bruts en silex noir de Tercis.

Effectif

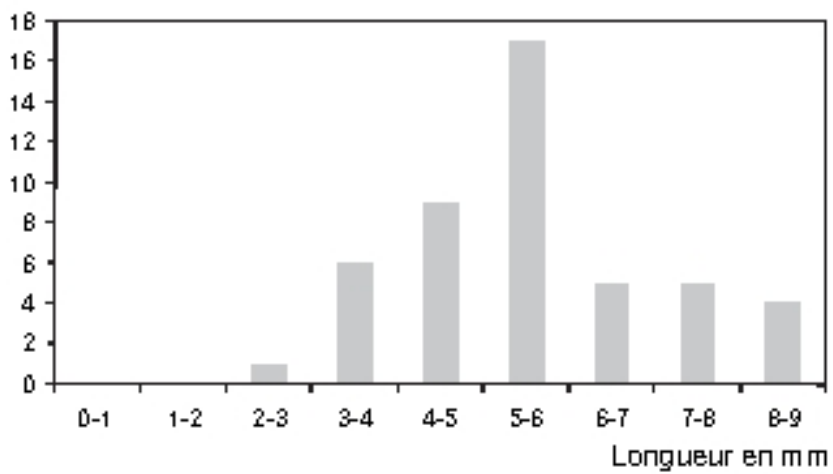


Figure 18 - Rapport largeur/épaisseur des 62 produits laminaires bruts de plein débitage en silex noir de Tercis.

Ėpaisseur en mm

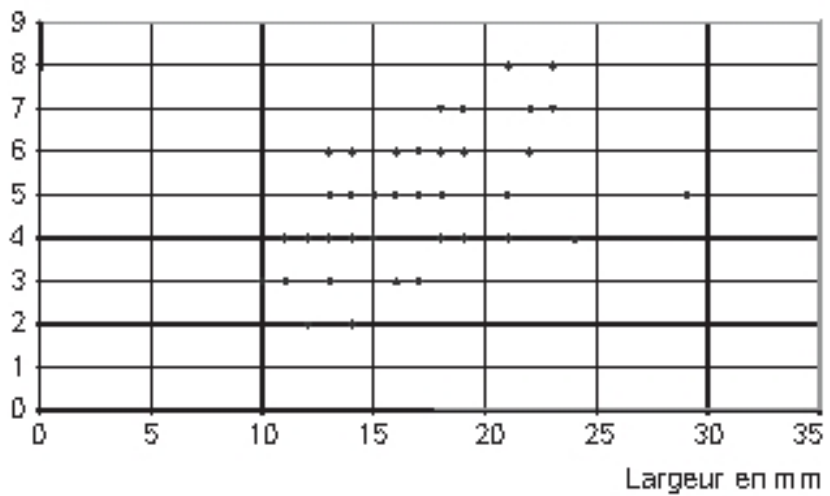

Figure 19 - Produits laminaires bruts de plein débitage portant des stigmates diagnostiques de I'utilisation d'une percussion tendre minérale. Photographie A. Simonet.
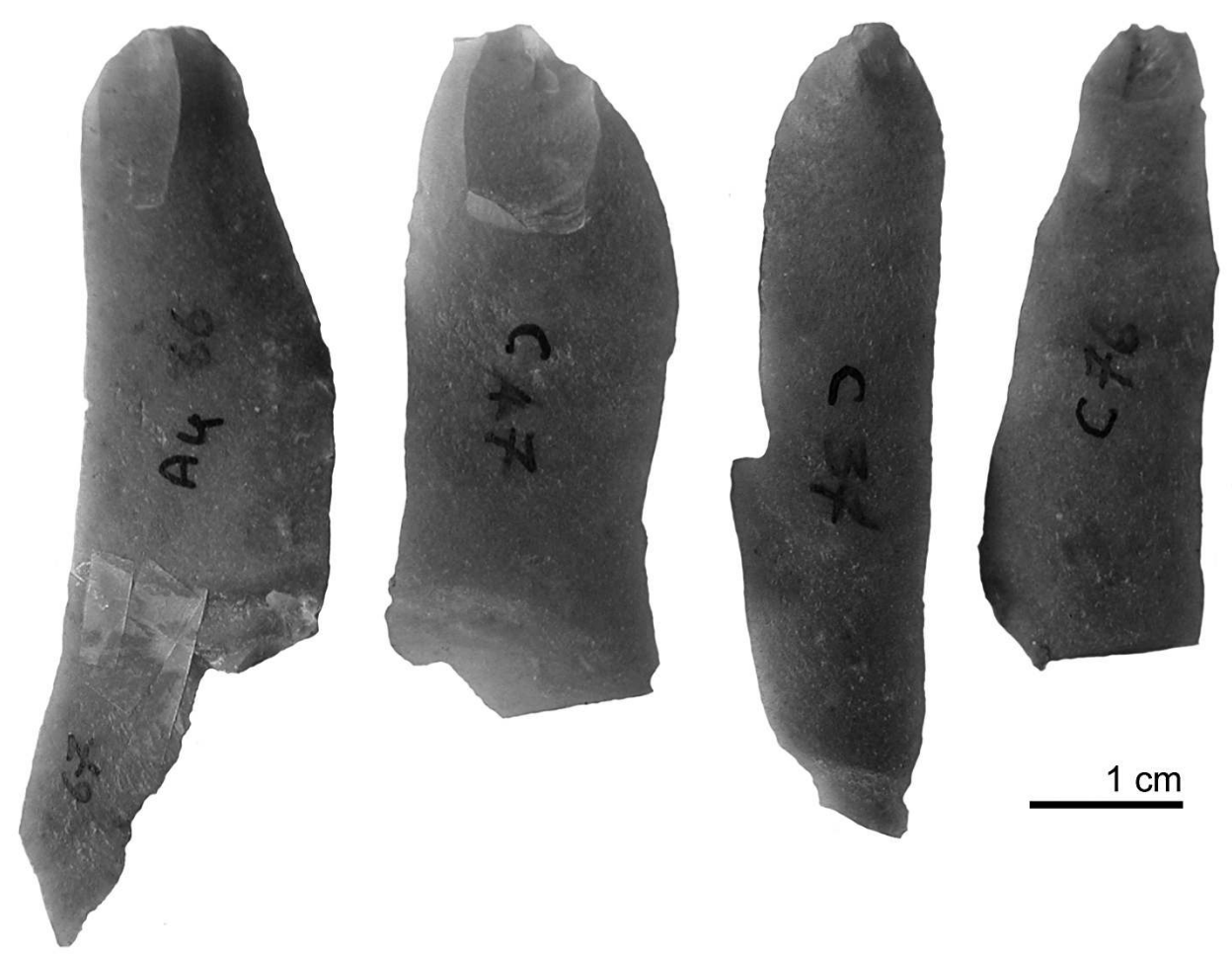

\section{4 - Conclusion et perspectives de recherche}

\section{1 - Tercis dans son contexte gravettien régional}

Il est remarquable de ne retrouver aucune pièce à dos aussi médiocre dans le Gravettien d'Isturitz ainsi que dans celui du Chantier I de Brassempouy (Simonet 2009, 2010, 2011, 2012). Certes, l'ancienneté des fouilles de la grotte d'Isturitz pourrait expliquer une telle absence mais les premières séries de tamisage n'ont pas révélé le moindre indice vers l'identification d'un façonnage d'armature par des apprentis. 
Inversement, les premières données de l'atelier de taille gravettien de Mugarduia Sur, en Espagne, corroborent l'existence d'une plus grande variabilité des chaînes opératoires au sein des sites spécialisés dans la taille du silex (Barandiarán et al. 2007 ; Simonet 2009). Ce site satellite des grands campements gravettiens de Brassempouy et d'Isturitz est le seul, avec Tercis, à présenter un nombre important de pièces à dos qui ne rentrent pas dans la typologie du Gravettien pyrénéen (Simonet 2010). Par conséquent, l'absence de témoins similaires aux pièces à dos maladroites de Tercis dans les grottes d'Isturitz et de Brassempouy permet d'envisager une interprétation sociologique. Dans cette hypothèse, l'apprentissage serait spatialement cloisonné, réservé à certains contextes comme les affleurements de matière première. Dans l'attente de nouvelles observations, nous proposons comme axe de recherche, d'interpréter la série de Tercis découverte par C. Normand comme l'une des plus anciennes traces découvertes d'un apprentissage encadré d'armatures lithiques, entre 29000 et 22000 ans avant le présent (fig. 20). Cette réflexion sur l'apprentissage présente de fortes implications paléo-sociologiques car elle offre un paramètre supplémentaire à prendre en compte dans la description d'une gestion complexe du territoire pyrénéen par les Gravettiens (Lacarrière et al. 2011 ; Redondo 2011 ; Simonet 2009, 2012).

\section{2 - Tercis dans son contexte gravettien européen}

L'apprentissage de la taille du silex renvoie à la question de la place des enfants et des adolescents dans les sociétés gravettiennes. Les données de Tercis s'insèrent dans un ensemble d'indices chronologiquement et géographiquement cohérents et provenant de sociétés gravettiennes ou contemporaines du Gravettien. Ces indices montrent que les jeunes sont, au cœur du Paléolithique supérieur, très tôt insérés dans les pratiques sociales. La grotte pyrénéenne de Gargas, par exemple, offre des représentations de mains négatives indiquant que les enfants participaient à certains rites et fréquentaient la grotte (Foucher et al. 2007). En République tchèque, l'étude des dermatoglyphes conservés sur les statuettes d'argile de Dolní Věstonice suggère que des enfants d'une dizaine d'années seraient les auteurs de certains modelages (Kralick et Novotny 2005). Mais la source documentaire la plus intéressante concerne sans doute les sépultures primaires réunissant dans un même espace le biologique et le culturel (Henry-Gambier 2008). L'analyse de la documentation anthropologique révèle ainsi, dès le début du Gravettien, des comportements nouveaux pour le Paléolithique supérieur européen : des défunts du stade périnatal à l'adolescence font l'objet d'un dépôt primaire intentionnel. Notons qu'à une date contemporaine du Gravettien, on retrouve des enfants inhumés à Sungir et à Mal'ta (Bader 1998; Cauwe et al. 1996; Derevianko et al. 1998). En définitive, l'ensemble de ces indices ne pourrait-il pas argumenter un changement de l'organisation sociale au Gravettien par rapport à l'Aurignacien antérieur, où se mettrait en place une dynamique de mise en valeur de la place des jeunes dans la société? 
Figure 20 - Tercis (Landes, France) : les vestiges archéologiques gravettiens d'un apprentissage encadré sur un atelier de taille il y a plus de 22000 ans? Dessin A. Simonet.

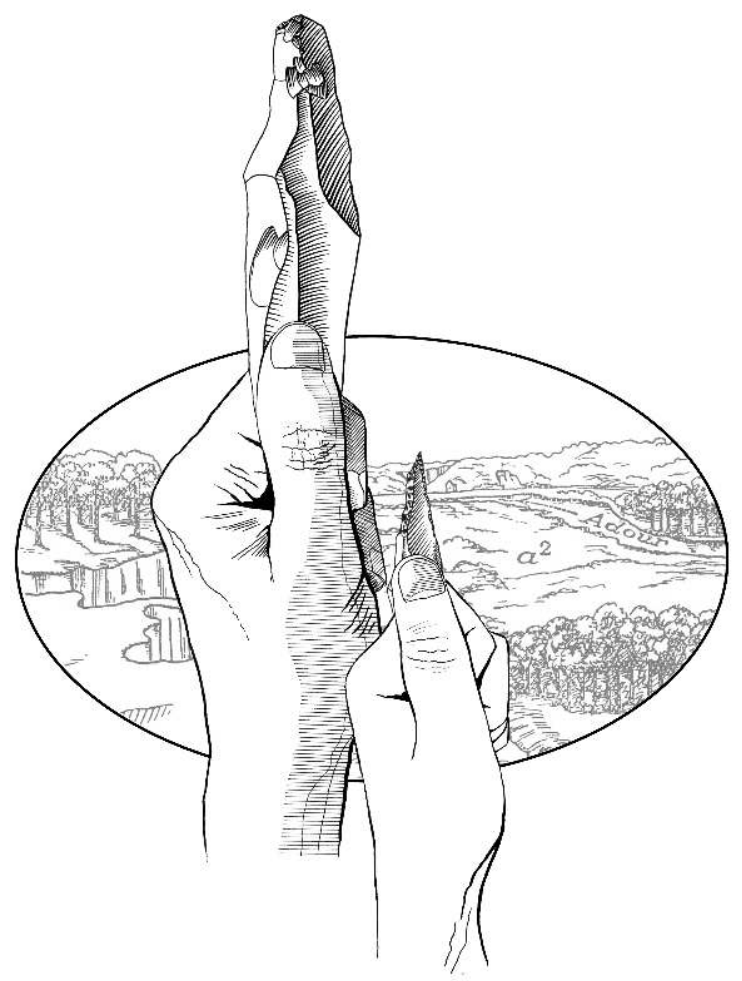

Nous adressons nos remerciements à $C$. Normand qui nous a confié l'étude de cette série dans le cadre de notre mémoire de maîtrise et à qui nous devons l'essentiel de notre connaissance du site de Tercis. Tous nos remerciements vont également à $F$. Bon qui a encadré et corrigé cette recherche universitaire.

\section{BIBLIOGRAPHIE}

ARAMBOUROU R. 1963 - Essai de paléogéographie du Paléolithique des Landes, Thèse complémentaire pour le Doctorat ès Sciences, Université de Bordeaux I.

BADER N. 1998 - Upper Palaeolithic site Sungir (graves and environment), Moscou, Scientific World, $270 \mathrm{p}$.

BARANDIARÁN I., BENEITEZ P., CAVA A., MILLÁN M. A. 2007 - El taller gravetiense de Mugarduia sur (Navarra) : identificación y cronología, Zephyrus, 60, p. 85-96.

BODU P. 1994 - Analyse typo-technologique du matériel lithique de quelques unités du site magdalénien de Pincevent (Seine-et-Marne). Applications spatiales, économiques et sociales, Thèse de doctorat de l'Université de Paris I Panthéon-Sorbonne. 3 vol.

CAUWE N., MEDVEDEV G., LIPNINA E., CLAES L., COUPE D., MODRIE S., MUXHARRAMOV S., OSADTSHY S., PETITT P., REBRIKOV P., ROGOVSKOÏ E., SULERJITSKY L., VOROBEVA G., 
XHENZIXHENOVA D. 1996 - Mal'ta en Sibérie : Présentation du programme de recherche des Musées royaux d'Art et d'Histoire de Bruxelles et de l'Université de l'Etat à Irkoutsk, Anthropologie et préhistoire, Bruxelles, Société royale belge d'anthropologie et de préhistoire, vol. 107, p. 109-130.

DAGUIN F. 1948 - L'Aquitaine occidentale, Géologie régionale de la France vol. 5, dir. Albert F. de Lapparent, Actualités scientifiques et industrielles 1050, Hermann \& Cie, Paris, 232 p.

DEREVIANKO A.P. et al. Ed. 1998 - The Paleolithic of Siberia : new discoveries and interpretations, Urbana, University of Illinois Press, $406 \mathrm{p}$.

DU BOUCHER H. 1877 - Les Aquenses primitifs ou Dax avant l'Histoire, Bulletin de la Société de Borda, p. 273-288 et 423-437.

DU BOUCHER H. 1878 - Quelques nouvelles trouvailles préhistoriques landaises, Bulletin de la Société de Borda, p. 56-61.

DU BOUCHER H. 1879 - Matériaux pour un catalogue des stations préhistoriques landaises. Bulletin de la Société de Borda, p. 307-317, Matériaux pour l'histoire primitive et naturelle de l'Homme, volume 14 , série $\mathrm{n}^{\circ} 2$, tome $\mathrm{X}$, p. 258-270.

FOUCHER P., SAN JUAN-FOUCHER C., RUMEAU Y. 2007 - La grotte de Gargas. Un siècle de découvertes, Édition Communauté de communes du canton de Saint-Laurent-de-Neste, 128 p.

HENRY-GAMBIER D. 2008 - Les sujets juvéniles du Paléolithique supérieur d'Europe à travers l'analyse des sépultures primaires : L'exemple de la culture gravettienne, In : Dr. F. Gusi, D. C. Olaria, L. S. Muriel. (eds), La muerte en la infancia, Servicio de Investigaciones Arqueologicas y Prehistoricas de la Diputacion de Castellon y el Laboratorio de Arqueologia Prehistorica de la Universidad « Jaume $1 »$ de Castellon, p. 331-364.

KARLIN C., PLOUX S., BODU P., PIGEOT N. 1993 - Some socio-economic aspects of the knapping process among groups of hunter-gatherers in the Paris Basin area. Colloque international, Versailles, 25-29 November 1988, Fondation Fyssen Symposium. L'usage de l'outil chez les primates humains et non-humains, Berthelet A. et Chavaillon J. (Ed.) Clarendon Press, Oxford, p. 318-337.

KAWALEK E. 2008 - L'atelier de taille de Tercis, Landes : tentative d'attribution chronoculturelle au sein de la séquence aurignacienne et réflexion sur le concept d'apprentissage, Mémoire de Master 1re année, Université de Toulouse II - Le Mirail, 125 p.

KLARIC L. 2006 - Comment interpréter la variabilité technique et qualitative des débitages lamellaires : les nucléus débités par méthode du Raysse à La Picardie (Indre-et-Loire, France), un « cas d'école » du Gravettien moyen d'Europe occidentale », In Aubry T., Almeida F., Araujo A. C., Tiffagom M. dir., Typologie vs Technologie, Résumés de la session C65, XVe congrès de l'UISPP, Lisbonne, 4-9 sept. 2006, p. 391.

KLARIC L. en préparation - New evidence of flint knapping apprenticeship for the Middle Stage of European Western Upper Palaeolithic : the case of Bladelets production at la Picardie (France).

KOZLOWSKI J. K., LENOIR M. 1988 - Analyse des pointes à dos des gisements périgordiens de l'Aquitaine, Uniwersytet Jagiellonski, Cracovie, $94 \mathrm{p}$.

KRALIK M., NOVOTNY V. 2005 - Dermatoglyphics on the ancient ceramics. In : Svoboda J.A. (ed.), Pavlov I Southeast. The Dolni Vestonice Studies, 14, p. 449-477.

LACARRIÈRE J., GOUTAS N., NORMAND C., SIMONET A. avec la collaboration de SCHWAB C. 2011 Vers une redéfinition des occupations gravettiennes de la grotte d'Isturitz (PyrénéesAtlantiques) : révision critique des collections « anciennes » par l'approche intégrée des données 
lithiques, fauniques et d'industrie osseuse, In Goutas N., Klaric L., Pesesse D., Guillermin P. dir., À la recherche des identités gravettiennes : actualités, questionnements et perspectives, Actes de la table ronde d'Aix-en-Provence, 2008, Société préhistorique française (« Mémoire » 52), p. 67-83.

MERLET J.C. 1996 - Le Périgordien supérieur et l'Azilien d'Arcet à Montaut. Archéologie des Pyrénées Occidentales et des Landes, tome 15, p. 119-126.

NORMAND C. 1987 - Le gisement paléolithique de plein air du Vignès à Tercis (Landes), Bulletin de la Société d'Anthropologie du Sud-Ouest, tome XXII, nº 2, p. 71-80.

NORMAND C. 1993 - Un atelier de taille de pièces à dos à Tercis (Landes), Archéologie des Pyrénées occidentales et des Landes, 1992/1993, tome 12, p. 27-51, 6 fig.

NORMAND C. 2002 - Les ressources en matières premières siliceuses dans la basse vallée de l'Adour et de ses affluents. Quelques données sur leur utilisation au paléolithique supérieur. In $\mathrm{N}$. Cazals dir., Comportements techniques et économiques des sociétés du Paléolithique supérieur dans le contexte pyrénéen. Projet collectif de recherche, p. 26-47.

PELEGRIN J. 1995 - Technologie lithique : le Châtelperronien de Roc-de-Combe (Lot) et de La Côte (Dordogne). Cahiers du Quaternaire, $n^{\circ} 20$, C.N.R.S., 297 p.

PELEGRIN J. 2000 - Les techniques de débitage laminaire au Tardiglaciaire : critère de diagnose et quelques réflexions, In : Valentin B., Bodu P. et Christensen M. (dir.) L'Europe centrale et septentrionale au Tardiglaciaire, Actes de la Table ronde internationale de Nemours, 13-16 mai 1997. Mémoires du Musée de Préhistoire d’Ile de France, 7, éd. A.P.R.A.I.F., Nemours, p. 73-86.

PIGEOT N. 1986 - Apprendre à débiter des lames : un cas d'éducation technique chez les Magdaléniens d'Etiolles, Bulletin de la Société Préhistorique Française, tome 83, p. 67-69.

PIGEOT N. 1988 - Apprendre à débiter des lames : un cas d'éducation technique chez des magdaléniens d'Étiolles, In : J. tixier (ed.), Technologie préhistorique. Paris, éditions du C.N.R.S., p. 63-70. (notes et monographies techniques du cra $\mathrm{n}^{\circ} 25$ ).

PIGEOT N. 1990 - Technical and Social actors in Prehistory : Flintknapping specialists and apprentices at Magdalenian Etiolles. Archaeological Review from Cambridge, 9, 1, n ${ }^{\circ}$ special, technology in the Humanities, p. 126-141.

PIGEOT N. dir. 2004 - Les derniers Magdaléniens d'Étiolles, Perspectives culturelles et paléohistoriques (l'unité d'habitation Q31). XXXVIIème supplément à Gallia Préhistoire, CNRS Editions, $351 \mathrm{p}$.

PLOUX S. 1989 - Approche archéologique de la variabilité des comportements techniques individuels : les tailleurs de l'unité 27-M89 de Pincevent, Thèse de Doctorat, Université de Paris X, 2 vol. , 584 p.

PLOUX S. 1991 - Technologie, technicité, techniciens : méthode de détermination d'auteurs et comportements techniques individuels, In 25 ans d'études technologiques en Préhistoire : bilan et perspectives. Actes des XIèmes rencontres internationales d'Archéologie et d'Histoire d'Antibes, 18-20 oct. 1990. Juan-les-Pins : APDCA, 1991, p. 201-214.

POTTIER R. 1872 - Etude préhistorique sur les environs de Dax (Landes), Matériaux pour l'histoire primitive et naturelle de l'Homme, volume 7, série n 2, p. 236-243.

REDONDO M. 2011 - L'atelier de taille de l'Avenue du Prissé, Bayonne (64). Approche techno-économique d'un site spécialisé gravettien dans le contexte pyrénéen, Mémoire de Master I, Université de Toulouse II - Le Mirail, 168 p.

SIMONET A. 2004 - L'atelier de taille gravettien de Tercis (Landes). Approche technologique, économique et sociologique : Étude de l'industrie lithique de deux concentrations dites « à pièces à dos » et « à grandes 
lames », Mémoire de Maîtrise, Université de Toulouse II - Le Mirail, 203 p., 93 planches et 14 photographies.

SIMONET A. 2008 - L'atelier de taille gravettien de Tercis (Landes) : un cas probable d'apprentissage de la confection d'armatures lithiques, In Pétillon J.-M., Dias-Meirinho M.-H., Cattelain P., Honegger M., Normand C., Valdeyron N. dir., Recherches sur les armatures de projectiles du Paléolithique supérieur au Néolithique, Actes du colloque C83, XVe congrès de l'UISPP, Lisbonne, 2006), P@lethnologie, 1, p. 184-211.

SIMONET A. 2009 - Les gravettiens des Pyrénées. Des armes aux sociétés, Thèse de doctorat, Université de Toulouse II - Le Mirail, 391 p.

SIMONET A. 2010 - Typologie des armatures lithiques gravettiennes de la grotte d'Isturitz (Pyrénées-Atlantiques, France), Oxford, Archaeopress (« BAR International Series » 2156), 133 p.

SIMONET A. 2011 - Le Gravettien du Chantier I de Brassempouy (Landes, France), In Goutas N., Klaric L., Pesesse D., Guillermin P. dir., À la recherche des identités gravettiennes : actualités, questionnements et perspectives, Actes de la table ronde d'Aix-en-Provence, 2008, Société préhistorique française (« Mémoire » 52), p. 57-66.

SIMONET A. 2012 - Brassempouy (Landes, France) ou la matrice gravettienne de l'Europe, Liège, ERAUL $133,141 \mathrm{p}$.

TEXIER J. P. 1993 - Géologie du site archéologique de Tercis, Archéologie des Pyrénées occidentales et des Landes, 1992/1993, tome 12, p. 53-59.

THIBAULT C. 1970 - Recherches sur les terrains quaternaires du Bassin de l'Adour, Thèse de Doctorat, Université de Bordeaux I, 4 vol. , 840 p., 171 fig. et LXVIII pl.

\section{RÉSUMÉS}

Le site archéologique de Tercis (Landes, France) est constitué d'un ensemble de petites concentrations de silex récoltées en surface depuis le XIXè siècle (fig. 1). La plupart des séries conséquentes ont été collectées par C. Normand à partir de la fin des années 1970 pendant l'exploitation d'une carrière de calcaire par la Société des Ciments de l'Adour (fig. 2). La délimitation spatiale de ces petites concentrations qui s'égrènent le long de la crête de Tercis renvoie à des activités rapides de taille du silex, exécutées en quelques heures, derniers témoins matériels d'installations éphémères liées à la richesse en silex brut offert par un anticlinal. De nombreux groupes humains ont ainsi été attirés par cette matière première depuis les phases anciennes du Paléolithique. Cependant, le site a été surtout fréquenté au Paléolithique supérieur, par les Gravettiens auxquels peut être attribuée la majeure partie de l'industrie lithique et notamment la petite série dont nous présentons l'étude. Elle provient d'une concentration qui, hélas, n'a pu être fouillée intégralement à cause du court délai imposé par la Société des Ciments de l'Adour (fig. 3 et tabl. 1). Conséquence logique du contexte d'atelier de taille, les outils sont minoritaires par rapport aux produits de débitage. Parmi les outils, les armatures sont largement dominantes (fig. 4, 5 et 6). L'originalité de cette série est de présenter un débitage maladroit auquel est associée une trentaine de pièces à dos de petites dimensions, inachevées et/ou ratées, dans un contexte d'atelier de taille pourtant paradoxalement propice au gaspillage. Dans le cadre de cet article, c'est vers une réflexion paléo-sociologique que nous souhaiterions aboutir à l'aide d'une présentation précise des données. En effet, à ces 34 pièces à dos irrégulières et/ou inachevées sont associés sept nucléus (fig. 7 à 14) dont un seul a été productif (fig. 7). Or, ce nucléus est également le seul exemplaire qui n'a pas été aménagé dans la variété du silex grisnoir de Tercis utilisé pour les autres nucléus et pour l'ensemble des pièces à dos. Par ailleurs, une 
centaine de produits laminaires relativement réguliers témoignent d'un débitage de supports laminaires rectilignes et réguliers, d'un gabarit très normé avec une longueur d'environ $60 \mathrm{~mm}$ pour une largeur de $15 \mathrm{~mm}$ et une épaisseur de $5 \mathrm{~mm}$ (fig. 16 à 19 et tabl. 2). Cette production est également attestée par un remontage d'un ensemble d'éclats et de produits laminaires qui en donnent une image en négatif (fig. 15). Néanmoins, les bons supports ne se retrouvent pas au sein des pièces à dos. Quelles raisons auraient alors motivé les tailleurs à exploiter des blocs et des éclats d'un silex médiocre et de petite dimension et à sélectionner les supports laminolamellaires les moins réguliers pour confectionner leurs pièces à dos ? En définitive, la singularité de cette série et l'ensemble des indices qu'elle permet de réunir se prêtent bien à la séduisante hypothèse de l'apprentissage (fig. 20) qui est jusqu'à présent archéologiquement très peu documentée (Pigeot 1988; Ploux 1991 ; Pelegrin 1995 ; Klaric 2006, en préparation). À défaut de pouvoir trancher d'une manière certaine en faveur de cette dernière, nous proposons d'alimenter les faits archéologiques encore trop restreints pour proposer une méthodologie pertinente de la restitution des gestes et des intentions.

1.Presentation of the site. The archaeological site of Tercis (Landes, France) contains several small superficial concentrations of lithic artefacts discovered since the XIX ${ }^{\circ}$ century (fig. 1 ). The majority of the substantial series were collected by C. Normand since the end of the 1970's during the open cast mining of a large limestone quarry by the "Société des Ciments de l'Adour" (fig. 2). The spatial delimitation of these small concentrations scattered along a one kilometre long crest of Tercis evokes quick activities, carried out in a few hours, remains witnesses of short-lived installations linked to the richness in flint offered by an anticline. Many human groups were thus attracted by this raw material since the old stages of Paleolithic. But the site knew its maximum frequentation during Upper Paleolithic by the gravettians to whom can be attributed the majority of lithic industries and notably the small assemblage which we present here.

2.Presentation of the assemblage with backed points. This lithic assemblage comes from a concentration which, unfortunately, could not have been completely collected because of the short time alloted for rescue excavations by the Société des Ciments de l'Adour (fig. 3 and tabl. 1). Logical consequence of workshop context, tools are minority compared to the flint knapping products. Among tools, lithic projectile weapon elements are largely dominant (fig. 4 to 6). The original aspects of this assemblage is to present a poorly executed flint reduction sequence associated with around thirty small backed pieces that are either unfinished and/or failed. These 34 unfinished and/or failed backed pieces are indeed associated to seven cores (fig. 7 to 14) the only one of which was productive (fig. 7). This core is also the only specimen which was not made in the variety of grey-black flint of Tercis used for other cores and the whole backed pieces. Moreover, a hundred relatively regular laminar blanks show a straight and regular blades production, with standardised dimensions around 2.5 inches long, 0.5 to 0.8 inches wide and 0.2 inches thick (fig. 16 to 19 and tabl. 2). This production is also attested by a refitting of several flakes and laminar blanks which give a negative picture of the intentions (fig. 15).

3. An example of projectile weapon elements apprenticeship? Within the framework of this article, we wish to head for a paleo-sociological reflexion relying on a precise presentation of the data. Indeed, this lithic assemblage shows several paradoxes. Firstly, knappers used low quality flint in a context of knapping workshop though paradoxically favourable for fine productions. Secondly, two types of flint exploitation can be noticed: on one side, an exploitation of good quality blocks of raw material which supplied good blanks as cores nos 1,8 and 9 and an important proportion of laminar blanks show it (fig. 7,15 and 16). On the other side, the majority of cores and products that refit to them testify of the complementary existence of the local exploitation of low quality blocks or flakes (fig. 8 to 14). Finally, straight and regular laminar blanks are not found within backed pieces. Then, which reason(s) would have motivated knappers to use low quality flint and to select the less regular blades and bladelets to make their 
backed pieces? The singularity and the whole clues offered by this assemblage lend themselves to the appealing hypothesis of apprenticeship (fig. 20) which is until now archaeologically poorly documented (Pigeot 1988 ; Ploux 1991 ; Pelegrin 1995 ; Klaric 2006, in preparation). Since we can't definitively break off in favour of this latter, we nevertheless propose to provide the archaeological facts still too restricted to ensure a relevant methodology of gestures and intentions restitution.

\section{INDEX}

Mots-clés : Tercis, atelier de taille, Gravettien, armature lithique, apprentissage

Keywords : Tercis, knapping workshop, Gravettian, projectile weapon element, apprenticeship

\section{AUTEUR}

\section{AURÉLIEN SIMONET}

TRACES - UMR 5608 - Maison de la Recherche, 5, allées A. Machado 31058 Toulouse Cedex 9 simonetaurelien@yahoo.fr 